\title{
Search for gravitational-wave bursts in LIGO data from the fourth science run
}

B Abbott ${ }^{1}$, R Abbott $^{1}$, R Adhikari $^{1}$, J Agresti $^{1}$, P Ajith $^{2}$, B Allen $^{2,3}$, R Amin ${ }^{4}$, S B Anderson ${ }^{1}$, W G Anderson ${ }^{3}$, M Arain ${ }^{5}$, M Araya ${ }^{1}$, H Armandula ${ }^{1}$, M Ashley ${ }^{6}$, S Aston ${ }^{7}$, P Aufmuth ${ }^{8}$, C Aulbert ${ }^{9}$, S Babak ${ }^{9}$, S Ballmer ${ }^{1}$, H Bantilan ${ }^{10}$, B C Barish ${ }^{1}$, C Barker ${ }^{11}$, D Barker ${ }^{11}$, B Barr ${ }^{12}$, P Barriga ${ }^{13}$, M A Barton ${ }^{12}$, K Bayer ${ }^{14}$, K Belczynski ${ }^{15}$, J Betzwieser ${ }^{14}$, P T Beyersdorf ${ }^{16}$, B Bhawal ${ }^{1}$, I A Bilenko ${ }^{17}$, G Billingsley $^{1}, \mathrm{R}_{\text {Biswas }}{ }^{3}$, E Black $^{1}$, K Blackburn ${ }^{1}$, L Blackburn ${ }^{4}$, D Blair ${ }^{13}$, B Bland ${ }^{11}$, J Bogenstahl ${ }^{12}$, L Bogue $^{18}$, R Bork ${ }^{1}, \mathbf{V}$ Boschi $^{1}, \mathbf{S}$ Bose $^{19}$, P R Brady ${ }^{1}$, V B Braginsky ${ }^{17}, \mathbf{J}$ E Brau ${ }^{20}$, M Brinkmann $^{2}$, A Brooks $^{21}$, D A Brown ${ }^{1,22}$, A Bullington ${ }^{23}$, A Bunkowski ${ }^{2}$, A Buonanno ${ }^{24}$, O Burmeister $^{2}$, D Busby $^{1}$, R L Byer ${ }^{23}$, L Cadonati ${ }^{14}$, G Cagnoli ${ }^{12}$, J B Camp ${ }^{25}$, J Cannizzo $^{25}$, $\mathrm{K}$ Cannon $^{3}$, C A Cantley ${ }^{12}, \mathrm{~J} \mathrm{Cao}^{14}$, L Cardenas ${ }^{1}$, M M Casey ${ }^{12}$, G Castaldi $^{26}$, C Cepeda ${ }^{1}$, E Chalkey ${ }^{12}$, P Charlton ${ }^{27}$, S Chatterji ${ }^{1}$, S Chelkowski ${ }^{2}$, Y Chen ${ }^{9}$, F Chiadini ${ }^{28}$, D Chin ${ }^{29}$, E Chin ${ }^{13}$, J Chow ${ }^{6}$, N Christensen ${ }^{10}$, J Clark ${ }^{12}$, P Cochrane ${ }^{2}$, T Cokelaer ${ }^{30}$, C N Colacino ${ }^{7}$,

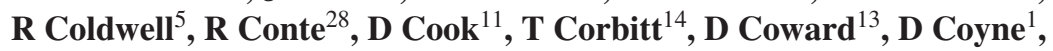
J D E Creighton ${ }^{3}$, T D Creighton ${ }^{1}$, R P Croce ${ }^{26}$, D R M Crooks ${ }^{12}$, A M Cruise ${ }^{7}$, A Cumming ${ }^{12}$, J Dalrymple ${ }^{31}$, E D'Ambrosio ${ }^{1}$, K Danzmann $^{2,8}$, G Davies $^{30}$, D DeBra $^{23}$, J Degallaix ${ }^{13}$, M Degree $^{23}$, T Demma $^{23}$, V Dergachev ${ }^{29}$, S Desai $^{32}$, R DeSalvo ${ }^{1}, \mathbf{S}_{\text {Dhurandhar }}{ }^{33}$, M Díaz ${ }^{34}$, J Dickson $^{6}$, A Di Credico ${ }^{31}$, G Diederichs $^{8}$, A Dietz $^{30}$, E E Doomes ${ }^{35}$, R W P Drever ${ }^{36}$, J-C Dumas ${ }^{13}$, R J Dupuis ${ }^{1}$, J G Dwyer ${ }^{37}$, P Ehrens $^{1}$, E Espinoza ${ }^{1}$, T Etzel $^{1}, \mathbf{M}_{\text {Evans }}{ }^{1}$, T Evans $^{18}$, S Fairhurst $^{1,30}$,

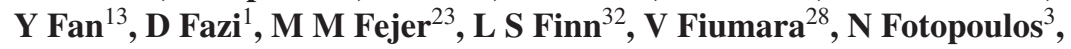

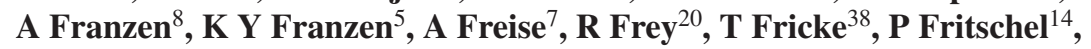
V V Frolov ${ }^{18}$, M Fyffe ${ }^{18}$, V Galdi ${ }^{26}$, J Garofoli ${ }^{11}$, I Gholami ${ }^{9}$, J A Giaime ${ }^{7,18}, \mathbf{S}$ Giampanis ${ }^{38}, \mathbf{K}$ D Giardina ${ }^{18}, \mathbf{K ~ G o d a}^{14}, \mathbf{E}$ Goetz $^{29}$, L M Goggin ${ }^{1}$, G González ${ }^{4}$, S Gossler ${ }^{6}$, A Grant ${ }^{12}$, S Gras ${ }^{13}$, C Gray ${ }^{11}$, M Gray $^{6}, \mathbf{J}$ Greenhalgh ${ }^{39}$, A M Gretarsson ${ }^{40}, \mathbf{R}$ Grosso ${ }^{34}$, H Grote $^{2}$, S Grunewald ${ }^{9}$, M Guenther ${ }^{11}$, R Gustafson ${ }^{29}$, B Hage $^{8}$, D Hammer ${ }^{3}$,

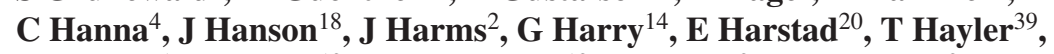
J Heefner ${ }^{1}$, I S Heng ${ }^{12}$, A Heptonstall ${ }^{12}$, M Heurs ${ }^{2}$, M Hewitson ${ }^{2}$, S Hild ${ }^{8}$, E Hirose $^{31}$, D Hoak ${ }^{18}$, D Hosken ${ }^{21}$, J Hough ${ }^{12}$, E Howell ${ }^{13}$, D Hoyland ${ }^{7}$, S H Huttner ${ }^{12}$, D Ingram ${ }^{11}$, E Innerhofer ${ }^{14}$, M Ito $^{20}$, Y Itoh $^{3}$, A Ivanov ${ }^{1}$, D Jackrel $^{23}$, B Johnson ${ }^{11}$, W W Johnson ${ }^{4}$, D I Jones ${ }^{41}$, G Jones ${ }^{30}$, R Jones $^{12}$, L Ju ${ }^{13}$, P Kalmus ${ }^{37}$, V Kalogera ${ }^{15}$, D Kasprzyk ${ }^{7}$, E Katsavounidis ${ }^{14}$, K Kawabe $^{11}$, S Kawamura ${ }^{42}$, F Kawazoe $^{42}$, W Kells ${ }^{1}$, D G Keppel $^{1}$, F Ya Khalili ${ }^{17}$, C Kim ${ }^{15}$, P King ${ }^{1}$, J S Kissel ${ }^{4}$, S Klimenko ${ }^{5}$, K Kokeyama ${ }^{42}$, V Kondrashov ${ }^{1}$, R K Kopparapu ${ }^{4}$, D Kozak ${ }^{1}$, B Krishnan ${ }^{9}$, P Kwee ${ }^{8}$, P K Lam ${ }^{6}$, M Landry ${ }^{11}$, B Lantz ${ }^{23}$, A Lazzarini ${ }^{1}$, 
B Lee ${ }^{13}$, M Lei $^{1}$, J Leiner $^{19}$, V Leonhardt ${ }^{42}$, I Leonor ${ }^{20}$, K Libbrecht $^{1}$, P Lindquist $^{1}$, N A Lockerbie ${ }^{43}$, M Longo ${ }^{28}$, M Lormand $^{18}$, M Lubinski $^{11}$, H Lück ${ }^{2,8}$, B Machenschalk ${ }^{9}$, M MacInnis ${ }^{14}$, M Mageswaran ${ }^{1}$,

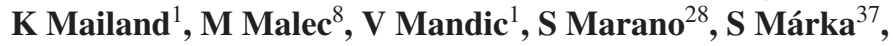
J Markowitz ${ }^{14}$, E Maros ${ }^{1}$, I Martin ${ }^{12}$, J N Marx ${ }^{12}$, K Mason ${ }^{14}$,

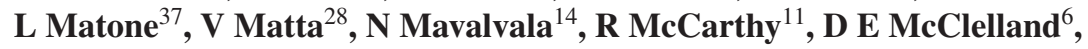
S C McGuire ${ }^{35}$, M McHugh ${ }^{44}$, K McKenzie ${ }^{6}$, J W C McNabb ${ }^{32}$, S McWilliams ${ }^{25}$, T Meier $^{8}$, A Melissinos ${ }^{38}$, G Mendell ${ }^{11}$, R A Mercer ${ }^{5}$, S Meshkov ${ }^{1}$, E Messaritaki ${ }^{1}$, C J Messenger ${ }^{12}$, D Meyers ${ }^{1}$, E Mikhailov ${ }^{14}$, S Mitra ${ }^{33}$, V P Mitrofanov ${ }^{17}$, G Mitselmakher ${ }^{5}$, R Mittleman ${ }^{14}$, O Miyakawa ${ }^{1}$, S Mohanty ${ }^{34}$, G Moreno ${ }^{11}$, K Mossavi ${ }^{2}$, C MowLowry ${ }^{6}$, A Moylan ${ }^{6}$, D Mudge ${ }^{21}$, G Mueller ${ }^{5}$, S Mukherjee ${ }^{34}, \mathbf{H}$ Müller-Ebhardt ${ }^{2}$, J Munch ${ }^{21}$, P Murray ${ }^{12}$, E Myers ${ }^{11}$, J Myers ${ }^{11}$, T Nash ${ }^{1}$, G Newton ${ }^{12}$, A Nishizawa ${ }^{42}$, K Numata ${ }^{25}$, B O'Reilly ${ }^{18}$, R O'Shaughnessy ${ }^{15}$, D J Ottaway ${ }^{14}$, H Overmier ${ }^{18}$, B J Owen ${ }^{32}$, Y Pan ${ }^{24}$, M A Papa ${ }^{3,9}$,

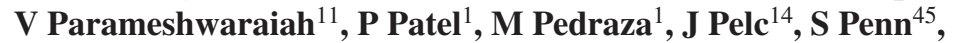
V Pierro ${ }^{26}$, I M Pinto ${ }^{26}$, M Pitkin $^{12}$, H Pletsch ${ }^{2}$, M V Plissi ${ }^{12}$, F Postiglione $^{28}$, R Prix ${ }^{9}$, V Quetschke ${ }^{5}$, F Raab ${ }^{11}$, D Rabeling ${ }^{6}$, H Radkins ${ }^{11}$, R Rahkola ${ }^{20}$, N Rainer $^{2}, M_{\text {Rakhmanov }}{ }^{32}$, M Ramsunder $^{32}$, K Rawlins ${ }^{14}$, S Ray-Majumder ${ }^{3}, \mathbf{V ~ R e}^{7}, \mathbf{H} \operatorname{Rehbein}^{2}$, S Reid ${ }^{12}$, D H Reitze ${ }^{5}$, L Ribichini ${ }^{2}$, R Riesen ${ }^{18}$, K Riles $^{29}$, B Rivera ${ }^{11}$, N A Robertson ${ }^{1,12}$, C Robinson ${ }^{30}$, E L Robinson ${ }^{7}$, S Roddy $^{18}$, A Rodriguez ${ }^{4}$, A M Rogan ${ }^{19}$, J Rollins ${ }^{37}$, J D Romano ${ }^{30}, \mathbf{J ~ R o m i e}^{18}$, R Route $^{23}, \mathbf{S}$ Rowan ${ }^{12}$, A Rüdiger ${ }^{2}$, L Ruet ${ }^{14}, \mathbf{P}$ Russell ${ }^{1}$, K Ryan $^{11}$, S Sakata ${ }^{42}$, M Samidi $^{1}$, L Sancho de la Jordana ${ }^{46}$, V Sandberg ${ }^{11}$, V Sannibale ${ }^{1}$, S Saraf ${ }^{47}$, P Sarin ${ }^{14}$, B S Sathyaprakash ${ }^{30}$, S Sato $^{42}$, P R Saulson ${ }^{31}$, R Savage ${ }^{11}$, P Savov ${ }^{22}$, S Schediwy ${ }^{13}$, R Schilling ${ }^{2}$, R Schnabel ${ }^{2}$, R Schofield ${ }^{20}$, B F Schutz ${ }^{9,30}$, P Schwinberg ${ }^{11}$, S M Scott ${ }^{6}$, A C Searle ${ }^{6}$, B Sears ${ }^{1}$, F Seifert ${ }^{2}$, D Sellers ${ }^{18}$, A S Sengupta ${ }^{30}$, P Shawhan ${ }^{24}$, D H Shoemaker ${ }^{14}$, A Sibley ${ }^{18}$, J A Sidles ${ }^{48}$, X Siemens ${ }^{1,22}$, D Sigg $^{11}$, S Sinha ${ }^{23}$, A M Sintes ${ }^{9,46}$, B J J Slagmolen ${ }^{6}$, J Slutsky ${ }^{4}$, J R Smith ${ }^{2}$, M R Smith ${ }^{1}$, K Somiya ${ }^{2,9}$, K A Strain ${ }^{12}$, D M Strom ${ }^{20}$, A Stuver ${ }^{32}$, T Z Summerscales ${ }^{49}$, K-X Sun ${ }^{23}$, M Sung $^{4}$, P J Sutton ${ }^{1}$, H Takahashi ${ }^{9}$, D B Tanner ${ }^{5}$, M Tarallo ${ }^{1}, \mathbf{R}$ Taylor $^{1}, \mathbf{R}$ Taylor $^{12}$, J Thacker ${ }^{18}$, K A Thorne ${ }^{32}$, K S Thorne ${ }^{22}$, A Thüring ${ }^{8}$, M Tinto ${ }^{1}$, K V Tokmakov ${ }^{12}$, C Torres ${ }^{34}$, C Torrie ${ }^{12}$, G Traylor ${ }^{18}$, M Trias ${ }^{46}$, W Tyler ${ }^{1}$, D Ugolini ${ }^{50}$, C Ungarelli ${ }^{7}$, K Urbanek $^{23}$, H Vahlbruch ${ }^{8}$, M Vallisneri ${ }^{22}$, C Van Den Broeck ${ }^{30}$, M Varvella ${ }^{1}$, S Vass $^{1}$, A Vecchio ${ }^{7}$, J Veitch $^{12}$, P Veitch ${ }^{21}$, A Villar ${ }^{1}$, C Vorvick ${ }^{11}$, S P Vyachanin ${ }^{17}$, S J Waldman ${ }^{1}$, L Wallace ${ }^{1}$, H Ward ${ }^{12}$, R Ward ${ }^{1}$, K Watts ${ }^{18}$, D Webber ${ }^{1}$, A Weidner ${ }^{2}$, M Weinert ${ }^{2}$, A Weinstein ${ }^{1}, \mathbf{R}$ Weiss ${ }^{14}, \mathbf{S}$ Wen $^{4}, \mathbf{K}$ Wette $^{6}$, J T Whelan ${ }^{9}$, D M Whitbeck ${ }^{32}$, S E Whitcomb ${ }^{1}$, B F Whiting ${ }^{5}$, C Wilkinson ${ }^{11}$, P A Willems ${ }^{1}$, L Williams ${ }^{5}$, B Willke ${ }^{2,8}$, I Wilmut ${ }^{39}$, W Winkler ${ }^{2}$, C C Wipf ${ }^{14}$, S Wise ${ }^{5}$, A G Wiseman ${ }^{3}$, G Woan ${ }^{12}$, D Woods ${ }^{3}$, R Wooley ${ }^{18}$, J Worden $^{11}, \mathrm{~W} \mathrm{Wu}^{5}$, I Yakushin ${ }^{18}, \mathrm{H}$ Yamamoto $^{1}, \mathrm{Z}$ Yan $^{13}$, S Yoshida ${ }^{51}$, N Yunes ${ }^{32}$, M Zanolin ${ }^{14}$, J Zhang ${ }^{29}$, L Zhang ${ }^{1}$, C Zhao ${ }^{13}$, N Zotov ${ }^{52}$, M Zucker ${ }^{14}$, H zur Mühlen ${ }^{8}$ and $\mathrm{J}_{\text {Zweizig }}{ }^{1}$

(LIGO Scientific Collaboration) 
${ }^{1}$ LIGO_California Institute of Technology, Pasadena, CA 91125, USA

2 Albert-Einstein-Institut, Max-Planck-Institut für Gravitationsphysik, D-30167 Hannover, Germany

${ }^{3}$ University of Wisconsin-Milwaukee, Milwaukee, WI 53201, USA

${ }^{4}$ Louisiana State University, Baton Rouge, LA 70803, USA

${ }^{5}$ University of Florida, Gainesville, FL 32611, USA

6 Australian National University, Canberra, 0200, Australia

${ }^{7}$ University of Birmingham, Birmingham, B15 2TT, UK

${ }^{8}$ Universität Hannover, D-30167 Hannover, Germany

${ }^{9}$ Albert-Einstein-Institut, Max-Planck-Institut für Gravitationsphysik, D-14476 Golm, Germany

${ }^{10}$ Carleton College, Northfield, MN 55057, USA

${ }^{11}$ LIGO Hanford Observatory, Richland, WA 99352, USA

12 University of Glasgow, Glasgow, G12 8QQ, UK

${ }^{13}$ University of Western Australia, Crawley, WA 6009, Australia

${ }^{14}$ LIGO_-Massachusetts Institute of Technology, Cambridge, MA 02139, USA

15 Northwestern University, Evanston, IL 60208, USA

16 San Jose State University, San Jose, CA 95192, USA

${ }^{17}$ Moscow State University, Moscow, 119992, Russia

${ }^{18}$ LIGO Livingston Observatory, Livingston, LA 70754, USA

${ }^{19}$ Washington State University, Pullman, WA 99164, USA

${ }^{20}$ University of Oregon, Eugene, OR 97403, USA

${ }^{21}$ University of Adelaide, Adelaide, SA 5005, Australia

22 Caltech-CaRT, Pasadena, CA 91125, USA

${ }^{23}$ Stanford University, Stanford, CA 94305, USA

${ }^{24}$ University of Maryland, College Park, MD 20742, USA

25 NASA/Goddard Space Flight Center, Greenbelt, MD 20771, USA

${ }^{26}$ University of Sannio at Benevento, I-82100 Benevento, Italy

27 Charles Sturt University, Wagga Wagga, NSW 2678, Australia

${ }^{28}$ University of Salerno, 84084 Fisciano (Salerno), Italy

${ }^{29}$ University of Michigan, Ann Arbor, MI 48109, USA

${ }^{30}$ Cardiff University, Cardiff, CF24 3AA, UK

${ }^{31}$ Syracuse University, Syracuse, NY 13244, USA

32 The Pennsylvania State University, University Park, PA 16802, USA

33 Inter-University Centre for Astronomy and Astrophysics, Pune-411007, India

34 The University of Texas at Brownsville and Texas Southmost College, Brownsville, TX 78520, USA

35 Southern University and A\&M College, Baton Rouge, LA 70813, USA

${ }^{36}$ California Institute of Technology, Pasadena, CA 91125, USA

37 Columbia University, New York, NY 10027, USA

${ }^{38}$ University of Rochester, Rochester, NY 14627, USA

${ }^{39}$ Rutherford Appleton Laboratory, Chilton, Didcot, Oxon OX11 0QX, UK

${ }^{40}$ Embry-Riddle Aeronautical University, Prescott, AZ 86301, USA

${ }^{41}$ University of Southampton, Southampton, SO17 1BJ, UK

${ }^{42}$ National Astronomical Observatory of Japan, Tokyo 181-8588, Japan

${ }^{43}$ University of Strathclyde, Glasgow, G1 1XQ, UK

${ }^{44}$ Loyola University, New Orleans, LA 70118, USA

45 Hobart and William Smith Colleges, Geneva, NY 14456, USA

${ }^{46}$ Universitat de les Illes Balears, E-07122 Palma de Mallorca, Spain

${ }^{47}$ Rochester Institute of Technology, Rochester, NY 14623, USA

${ }^{48}$ University of Washington, Seattle, WA 98195 , USA

${ }^{49}$ Andrews University, Berrien Springs, MI 49104, USA

${ }^{50}$ Trinity University, San Antonio, TX 78212, USA

${ }^{51}$ Southeastern Louisiana University, Hammond, LA 70402, USA

52 Louisiana Tech University, Ruston, LA 71272, USA

E-mail: pshawhan@umd.edu

Received 3 May 2007, in final form 28 August 2007

Published 24 October 2007

Online at stacks.iop.org/CQG/24/5343 


\begin{abstract}
The fourth science run of the LIGO and GEO 600 gravitational-wave detectors, carried out in early 2005 , collected data with significantly lower noise than previous science runs. We report on a search for short-duration gravitationalwave bursts with arbitrary waveform in the $64-1600 \mathrm{~Hz}$ frequency range appearing in all three LIGO interferometers. Signal consistency tests, data quality cuts and auxiliary-channel vetoes are applied to reduce the rate of spurious triggers. No gravitational-wave signals are detected in 15.5 days of live observation time; we set a frequentist upper limit of 0.15 day $^{-1}$ (at $90 \%$ confidence level) on the rate of bursts with large enough amplitudes to be detected reliably. The amplitude sensitivity of the search, characterized using Monte Carlo simulations, is several times better than that of previous searches. We also provide rough estimates of the distances at which representative supernova and binary black hole merger signals could be detected with $50 \%$ efficiency by this analysis.
\end{abstract}

PACS numbers: $04.80 . \mathrm{Nn}, 95.75 .-\mathrm{z}, 95.85 . \mathrm{Sz}$

\title{
1. Introduction
}

Large interferometers are now being used to search for gravitational waves with sufficient sensitivity to be able to detect signals from distant astrophysical sources. At present, the three detectors of the Laser Interferometer Gravitational-Wave Observatory (LIGO) project [1] have achieved strain sensitivities consistent with their design goals, while the GEO 600 [2] and Virgo [3] detectors are in the process of being commissioned and are expected to reach comparable sensitivities. Experience gained with these detectors, TAMA300 [4], and several small prototype interferometers has nurtured advanced designs for future detector upgrades and new facilities, including Advanced LIGO [5], Advanced Virgo [6] and the Large-scale Cryogenic Gravitational-Wave Telescope (LCGT) proposed to be constructed in Japan [7]. The LIGO Scientific Collaboration (LSC) carries out the analysis of data collected by the LIGO and GEO 600 gravitational-wave detectors, and has begun to pursue joint searches with other collaborations (see, for example, [8]) as the network of operating detectors evolves.

As the exploration of the gravitational-wave sky can now be carried out with greater sensitivity than ever before, it is important to search for all plausible signals in the data. In addition to well modeled signals such as those from binary inspirals [9] and spinning neutron stars [10], some astrophysical systems may emit gravitational waves which are modeled imperfectly (if at all) and therefore cannot reliably be searched for using matched filtering. Examples of such imperfectly-modeled systems include binary mergers (despite recent advances in the fidelity of numerical relativity calculations for at least some cases; see, for example, [11]) and stellar core collapse events. For the latter, several sets of simulations have been carried out in the past (see, for example, $[12,13]$ ), but more recent simulations have suggested a new resonant core oscillation mechanism, driven by in-falling material, which appears to power the supernova explosion and also to emit strong gravitational waves $[14,15]$. Given the current uncertainties regarding gravitational-wave emission by systems such as these, as well as the possibility of detectable signals from other astrophysical sources which are unknown or for which no attempt has been made to model gravitational-wave emission, it is desirable to cast a wide net. 
In this paper, we report the results of a search for gravitational-wave 'bursts' that is designed to be able to detect short-duration ( $\ll 1 \mathrm{~s})$ signals of arbitrary form as long as they have significant signal power in the most sensitive frequency band of LIGO, considered here to be $64-1600 \mathrm{~Hz}$. This analysis uses LIGO data from the fourth science run carried out by the LSC, called S4, and uses the same basic methods as previous LSC burst searches $[17,18]$ that were performed using data from the S2 and S3 science runs. (A burst search was performed using data from the $\mathrm{S} 1$ science run using different methods [16].) We briefly describe the instruments and data collection in section 2. In sections 3 and 4, we review the two complementary signal processing methods-one based on locating signal power in excess of the baseline noise and the other based on cross-correlating data streams-that are used together to identify gravitational-wave event candidates. We note where the implementations have been improved relative to the earlier searches and describe the signal consistency tests which are based on the outputs from these tools. Section 5 describes additional selection criteria which are used to 'clean up' the data sample, reducing the average rate of spurious triggers in the data. The complete analysis 'pipeline' finds no event candidates that pass all of the selection criteria, so we present in section 6 an upper limit on the rate of gravitational-wave events which would be detected reliably by our pipeline.

The detectability of a given type of burst, and thus the effective rate limit for a particular astrophysical source model, depends on the signal waveform and amplitude; in general, the detection efficiency (averaged over sky positions and arrival times) is less than unity. We do not attempt a comprehensive survey of possible astrophysical signals in this paper, but use a Monte Carlo method with a limited number of ad hoc simulated signals to evaluate the amplitude sensitivity of our pipeline, as described in section 7 . Overall, this search has much better sensitivity than previous searches, mostly due to using lower-noise data and partly due to improvements in the analysis pipeline. In section 8 , we estimate the amplitude sensitivity for certain modeled signals of interest and calculate approximate distances at which those signals could be detected with 50\% efficiency. This completed S4 search sets the stage for burst searches now underway using data from the S5 science run of the LIGO and GEO 600 detectors, which benefit from much longer observation time and will be able to detect even weaker signals.

\section{Instruments and data collection}

LIGO comprises two observatory sites in the United States with a total of three interferometers. As shown schematically in figure 1, the optical design is a Michelson interferometer augmented with additional partially-transmitting mirrors to form Fabry-Perot cavities in the arms and to 'recycle' the outgoing beam power by interfering it with the incoming beam. Servo systems are used to 'lock' the mirror positions to maintain resonance in the optical cavities, as well as to control the mirror orientations, laser frequency and intensity and many other degrees of freedom of the apparatus. Interference between the two beams recombining at the beam splitter is detected by photodiodes, providing a measure of the difference in arm lengths that would be changed by a passing gravitational wave. The large mirrors which direct the laser beams are suspended from wires, with the support structures isolated from ground vibrations using stacks of inertial masses linked by damped springs. Active feed-forward and feedback systems provide additional suppression of ground vibrations for many of the degrees of freedom. The beam path of the interferometer, excluding the laser light source and the photodiodes, is entirely enclosed in a vacuum system. The LIGO Hanford Observatory in Washington state has two interferometers within the same vacuum system, one with arms $4 \mathrm{~km}$ long (called H1) 


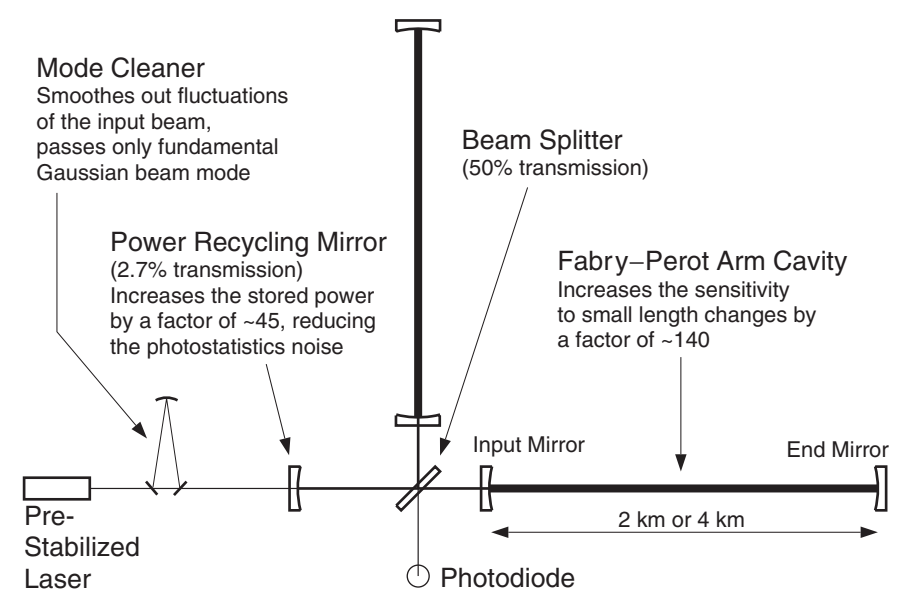

Figure 1. Simplified optical layout of a LIGO interferometer.

and the other with arms $2 \mathrm{~km}$ long (called H2). The LIGO Livingston Observatory in Louisiana has a single interferometer with $4 \mathrm{~km}$ long arms, called L1.

The response of an interferometer to a gravitational wave arriving at local time $t$ depends on the dimensionless strain amplitude and polarization of the wave and its arrival direction with respect to the arms of the interferometer. In the low-frequency limit, the differential strain signal detected by the interferometer (effective arm length difference divided by the length of an arm) can be expressed as a projection of the two polarization components of the gravitational wave, $h_{+}(t)$ and $h_{\times}(t)$, with antenna response factors $F_{+}(\alpha, \delta, t)$ and $F_{\times}(\alpha, \delta, t)$ :

$$
h_{\text {det }}(t)=F_{+}(\alpha, \delta, t) h_{+}(t)+F_{\times}(\alpha, \delta, t) h_{\times}(t),
$$

where $\alpha$ and $\delta$ are the right ascension and declination of the source. $F_{+}$and $F_{\times}$are distinct for each interferometer site and change slowly with $t$ over the course of a sidereal day as Earth's rotation changes the orientation of the interferometer with respect to the source location.

The electrical signal from the photodiode is filtered and digitized continuously at a rate of $16384 \mathrm{~Hz}$. The time series of digitized values, referred to as the 'gravitational-wave channel' (GW channel), is recorded in a computer file, along with a timestamp derived from the Global Positioning System (GPS) and additional information. The relationship between a given gravitational-wave signal and the digitized time series is measured in situ by imposing continuous sinusoidal position displacements of known amplitude on some of the mirrors. These are called 'calibration lines' because they appear as narrow line features in a spectrogram of the GW channel.

Commissioning the LIGO interferometers has required several years of effort and was the primary activity through late 2005. Beginning in 2000, a series of short data collection runs was begun to establish operating procedures, test the detector systems with stable configurations and provide data for the development of data analysis techniques. The first data collection run judged to have some scientific interest, science run S1, was conducted in August-September 2002 with detector noise more than two orders of magnitude higher than the design goal. Science runs S2 and S3 followed in 2003 with steadily improving detector noise, but with a poor duty cycle for L1 due primarily to low-frequency, large-amplitude ground motion from human activities and weather. During 2004, a hydraulic pre-isolation system was installed and commissioned at the Livingston site to measure the ground motion and counteract it with 


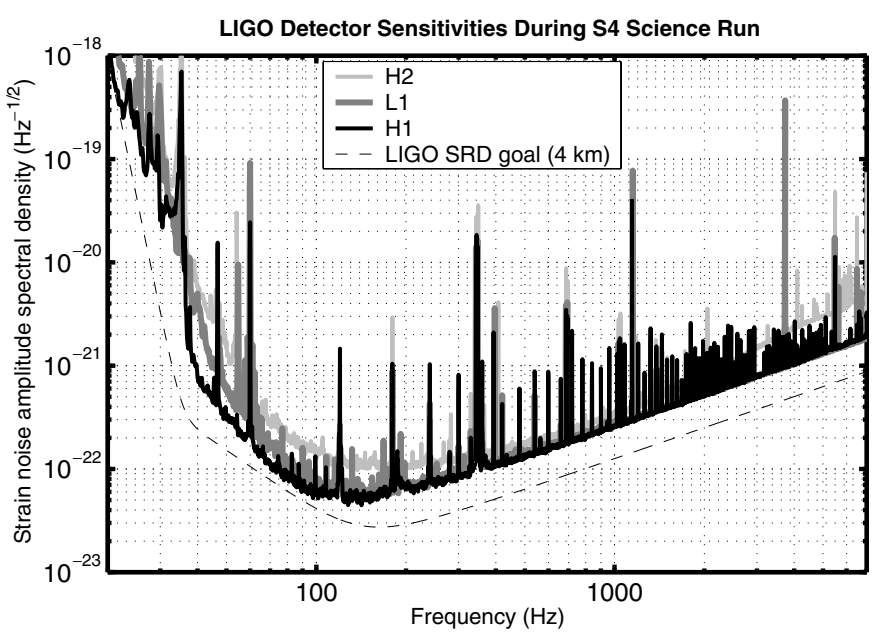

Figure 2. Best achieved detector noise for the three LIGO interferometers during the S4 science run, in terms of equivalent gravitational-wave strain amplitude spectral density. 'LIGO SRD goal' is the sensitivity goal for the 4-km LIGO interferometers set forth in the 1995 LIGO Science Requirements Document [19].

a relative displacement between the external and internal support structures for the optical components, keeping the internal components much closer to an inertial frame at frequencies above $0.1 \mathrm{~Hz}$. At the same time, several improvements were made to the $\mathrm{H} 1$ interferometer at Hanford to allow the laser power to be increased to the full design power of $10 \mathrm{~W}$.

The S4 science run, which lasted from 22 February to 23 March 2005, featured good overall 'science-mode' duty cycles of $80.5 \%, 81.4 \%$ and $74.5 \%$ for $\mathrm{H} 1, \mathrm{H} 2$ and L1, respectively, corresponding to observation times of 570, 576 and $528 \mathrm{~h}$. Thanks to the improvements made after the S3 run, the detector noise during S4 was within a factor of two of the design goal over most of the frequency band, as shown in figure 2. The GEO 600 interferometer also collected data throughout the S4 run, but was over a factor of 100 less sensitive than the LIGO interferometers at $200 \mathrm{~Hz}$ and a factor of few at and above the $1 \mathrm{kHz}$ frequency range. The analysis approach used in this paper effectively requires a gravitationalwave signal to be distinguishable above the noise in each of a fixed set of detectors, so it uses only the three LIGO interferometers and not the GEO 600. There are a total of $402 \mathrm{~h}$ of S4 during which all three LIGO interferometers were simultaneously collecting science-mode data.

\section{Trigger generation}

The first stage of the burst search pipeline is to identify times when the GW channels of the three interferometers appear to contain signal power in excess of the baseline noise; these times, along with parameters derived from the data, are called 'triggers' and are used as input to later processing stages. As in previous searches [17, 18], the WaveBurst algorithm [20] is used for this purpose; it will only be summarized here [21].

WaveBurst performs a linear wavelet packet decomposition, using the symlet wavelet basis [22], on short intervals of gravitational-wave data from each interferometer. This decomposition produces a time-frequency map of the data similar to a windowed Fourier 
transformation. A time-frequency data sample is referred to as a pixel. Pixels containing significant excess signal power are selected in a non-parametric way by ranking them with other pixels at nearby times and frequencies. As in the S3 analysis, WaveBurst has been configured for $\mathrm{S} 4$ to use six different time resolutions and corresponding frequency resolutions, ranging from $1 / 16 \mathrm{~s}$ by $8 \mathrm{~Hz}$ to $1 / 512 \mathrm{~s}$ by $256 \mathrm{~Hz}$, to be able to closely match the natural timefrequency properties of a variety of burst signals. The wavelet decomposition is restricted to 64-2048 Hz. At any given resolution, significant pixels from the three detector data streams are compared and coincident pixels are selected; these are used to construct 'clusters', potentially spanning many pixels in time and/or frequency, within which there is evidence for a common signal appearing in the different detector data streams. These coincident clusters form the basis for triggers, each of which is characterized by a central time, duration, central frequency, frequency range and overall significance $Z_{\mathrm{g}}$ as defined in [23]. $Z_{\mathrm{g}}$ is calculated from the pixels in the cluster and is roughly proportional to the geometric average of the excess signal power measured in the three interferometers, relative to the average noise in each interferometer at the relevant frequency. Thus, a large value of $Z_{\mathrm{g}}$ indicates that the signal power in those pixels is highly unlikely to have resulted from usual instrumental noise fluctuations. In addition, the absolute strength of the signal detected by each interferometer within the sensitive frequency band of the search is estimated in terms of the root-sum-squared amplitude of the detected strain,

$$
h_{\mathrm{det}}^{\mathrm{rss}}=\sqrt{\int\left|h_{\mathrm{det}}(t)\right|^{2} \mathrm{~d} t} .
$$

WaveBurst was run on time intervals during which all three LIGO interferometers were in science mode, but omitting periods when simulated signals were injected into the interferometer hardware, any photodiode readout experienced an overflow, or the data acquisition system was not operating. In addition, the last $30 \mathrm{~s}$ of each science-mode data segment were omitted because it was observed that loss of 'lock' is sometimes preceded by a period of instability. These selection criteria reduced the amount of data processed by WaveBurst from $402 \mathrm{~h}$ to $391 \mathrm{~h}$.

For this analysis, triggers found by WaveBurst are initially required to have a frequency range which overlaps $64-1600 \mathrm{~Hz}$. An initial significance cut, $Z_{\mathrm{g}} \geqslant 6.7$, is applied to reject the bulk of the triggers and limit the number passed along to later stages of the analysis. Figure 3 shows the distribution of $Z_{\mathrm{g}}$ prior to applying this significance cut.

Besides identifying truly simultaneous signals in the three data streams, WaveBurst applies the same pixel matching and cluster coincidence tests to the three data streams with many discrete relative time shifts imposed between the Hanford and Livingston data streams, each much larger than the maximum light travel time between the sites and the duration of the signals targeted by this search. The time-shifted triggers found in this way provide a large sample to allow the 'background' (spurious triggers produced in response to detector noise in the absence of gravitational waves) to be studied, under the assumption that the detector noise properties do not vary much over the span of a few minutes and are independent at the two sites. The two Hanford data streams are not shifted relative to one another, so that any local environmental effects which influence both detectors are preserved. In fact, some correlation in time is observed between noise transients in the $\mathrm{H} 1$ and $\mathrm{H} 2$ data streams.

Initially, WaveBurst found triggers for 98 time shifts in multiples of $3.125 \mathrm{~s}$ between -156.25 and $-6.25 \mathrm{~s}$ and between +6.25 and $+156.25 \mathrm{~s}$. These 5119 triggers, called the 'tuning set', were used to choose the parameters of the signal consistency tests and additional selection criteria described in the following two sections. As shown in figure 4, the rate of triggers in the tuning set is roughly constant for all time shifts, with a marginal $\chi^{2}$ value 


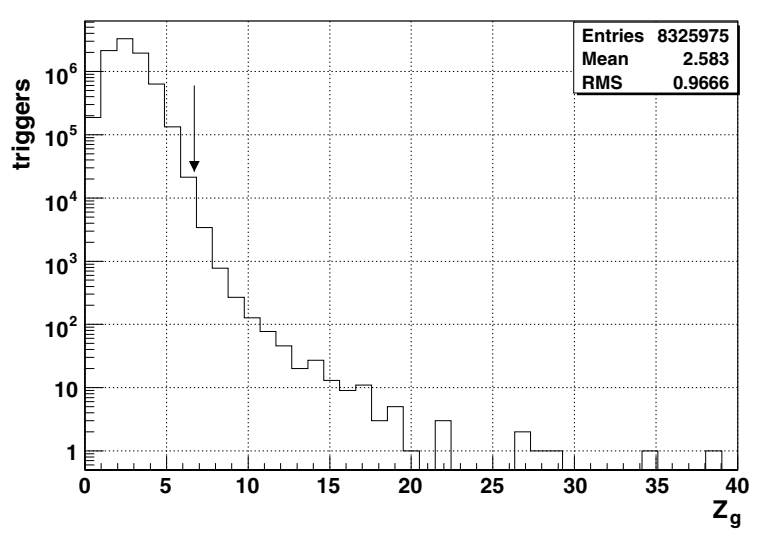

Figure 3. Distribution of $Z_{\mathrm{g}}$ values for all WaveBurst triggers. The arrow shows the location of the initial significance cut, $Z_{\mathrm{g}}>6.7$.

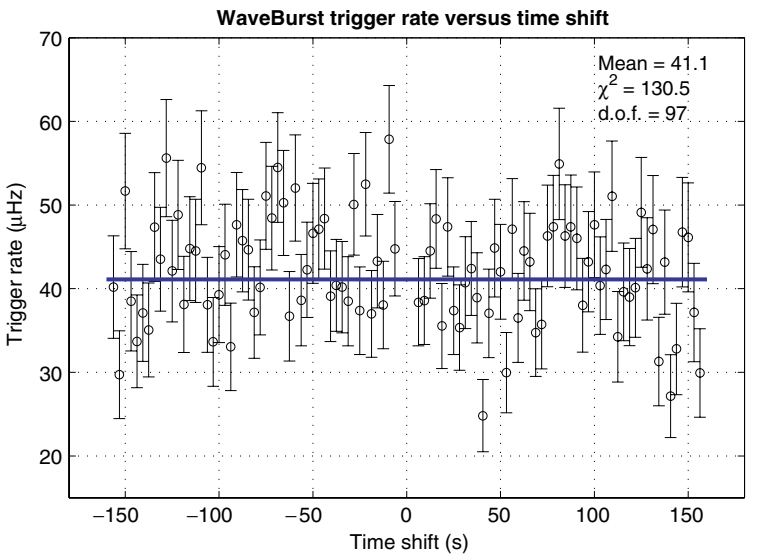

Figure 4. WaveBurst trigger rate as a function of the relative time shift applied between the Hanford and Livingston data streams. The horizontal line is a fit to a constant value, yielding a $\chi^{2}$ of 130.5 for 97 degrees of freedom.

but without any gross dependence on time shift. The unshifted triggers were kept hidden throughout the tuning process, in order to avoid the possibility of human bias in the choice of analysis parameters.

\section{Signal consistency tests}

The WaveBurst algorithm requires only a rough consistency among the different detector data streams-namely some apparent excess power in the same pixels in the wavelet decomposition - to generate a trigger. This section describes more sophisticated consistency tests based on the detailed content of the GW channels. These tests succeed in eliminating most WaveBurst triggers in the data, while keeping essentially all triggers generated in response to simulated gravitational-wave signals added to the data streams. (The simulation method is described in section 7.) Similar tests were also used in the S3 search [18]. 


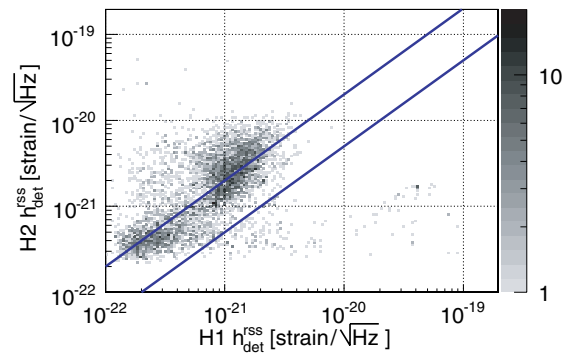

(a)

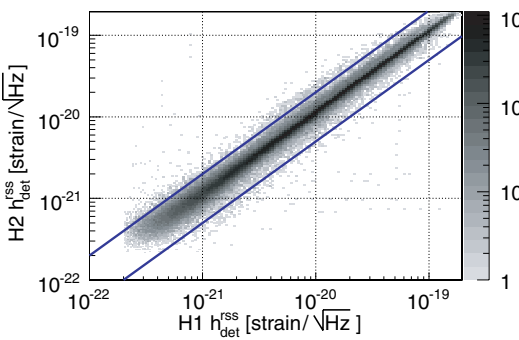

(b)

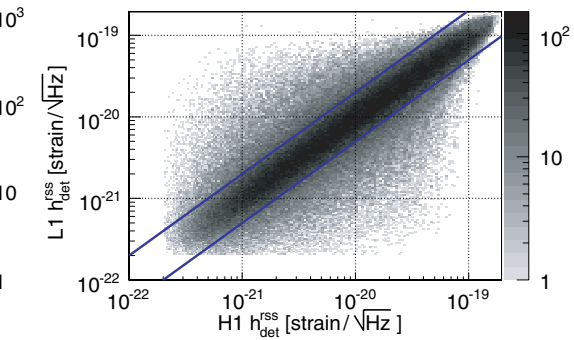

(c)

Figure 5. (a) A two-dimensional histogram, with bin count indicated by grayscale, of $\mathrm{H} 2$ versus H1 amplitudes reconstructed by WaveBurst for the tuning set of time-shifted triggers. (b) A two-dimensional histogram of $\mathrm{H} 2$ versus $\mathrm{H} 1$ amplitudes reconstructed for simulated sine-Gaussian signals with a wide range of frequencies and amplitudes from sources uniformly distributed over the sky (see section 7). In these plots, the diagonal lines show the limits of the $\mathrm{H} 1 / \mathrm{H} 2$ amplitude consistency cut: $0.5<$ ratio $<2$. (c) A two-dimensional histogram of L1 versus H1 amplitudes for the same simulated sine-Gaussian signals. Diagonal lines are drawn at ratios of 0.5 and 2 only to guide the eye; no cut is applied using this pair of interferometers.

\subsection{H1/H2 amplitude consistency test}

Because the two Hanford interferometers are co-located and co-aligned, they will respond identically (in terms of strain) to any given gravitational wave. Thus, the overall root-sumsquared amplitudes of the detected signals, estimated by WaveBurst according to equation (2), should agree well if the estimation method is reliable. Figure 5(a) shows that the time-shifted triggers in the tuning set often have poor agreement between the detected signal amplitudes in $\mathrm{H} 1$ and H2. In contrast, simulated signals injected into the data are found with amplitudes which usually agree within a factor of 2 , as shown in figure $5(b)$. Therefore, we keep a trigger only if the ratio of estimated signal amplitudes is in the range 0.5-2.

The Livingston interferometer is roughly aligned with the Hanford interferometers, but the curvature of the Earth makes exact alignment impossible. The antenna responses to a given gravitational wave will tend to be similar, but not reliably enough to allow a consistency test which is both effective at rejecting noise triggers and efficient at retaining simulated signals, as shown in figure $5(c)$.

\subsection{Cross-correlation consistency tests}

The amplitude consistency test described in the previous subsection simply compares scalar quantities derived from the data, without testing whether the waveforms are similar in detail. 
We use a program called CorrPower [24], also used in the S3 burst search [18], to calculate statistics based on Pearson's linear correlation statistic,

$$
r=\frac{\sum_{i=1}^{N}\left(x_{i}-\bar{x}\right)\left(y_{i}-\bar{y}\right)}{\sqrt{\sum_{i=1}^{N}\left(x_{i}-\bar{x}\right)^{2}} \sqrt{\sum_{i=1}^{N}\left(y_{i}-\bar{y}\right)^{2}}} .
$$

In the above expression $\left\{x_{i}\right\}$ and $\left\{y_{i}\right\}$ are sequences selected from the two GW channel time series, possibly with a relative time shift, and $\bar{x}$ and $\bar{y}$ are their respective mean values. The length of each sequence, $N$ samples, corresponds to a chosen time window (see below) over which the correlation is to be evaluated. $r$ assumes values between -1 for fully anti-correlated sequences and +1 for fully correlated sequences.

The $r$ statistic measures the correlation between two data streams, such as would be produced by a common gravitational-wave signal embedded in uncorrelated detector noise [25]. It compares waveforms without being sensitive to the relative amplitudes, and is thus complementary to the $\mathrm{H} 1 / \mathrm{H} 2$ amplitude consistency test described above. Furthermore, the $r$ statistic may be used to test for a correlation between $\mathrm{H} 1$ and $\mathrm{L} 1$ or between $\mathrm{H} 2$ and L1, even though these pairs consist of interferometers with different antenna response factors, because each polarization component will produce a measurable correlation for a suitable relative time delay (unless the wave happens to arrive from one of the special directions for which one of the detectors has a null response for that polarization component). In the special case of a linearly polarized gravitational wave, the detected signals will simply differ by a multiplicative factor, which can be either positive or negative depending on the polarization angle and arrival direction.

Before calculating the $r$ statistic for each detector pair, the data streams are filtered to select the frequency band of interest (bandpass between $64 \mathrm{~Hz}$ and $1600 \mathrm{~Hz}$ ) and whitened to equalize the contribution of noise from all frequencies within this band. The filtering is the same as was used in the S3 search [18] except for the addition of a $Q=10$ notch filter, centered at $345 \mathrm{~Hz}$, to avoid measuring correlations from the prominent vibrational modes of the wires used to suspend the mirrors, which are clustered around that frequency. The $r$ statistic is then calculated over multiple time windows with lengths of 20,50 and $100 \mathrm{~ms}$ and a range of starting times, densely placed (99\% overlap) to cover the full duration of the trigger as reported by WaveBurst; the maximum value from among these different time windows is used.

CorrPower [26] calculates two quantities, derived from the $r$ statistic, which are used to select triggers. The first of these, called $R_{0}$, is simply the signed cross-correlation between $\mathrm{H} 1$ and $\mathrm{H} 2$ with no relative time delay. Triggers with $R_{0}<0$ are rejected. The second quantity, called $\Gamma$, combines the $r$-statistic values from the three detector pairs, allowing relative time delays of up to $11 \mathrm{~ms}$ between $\mathrm{H} 1$ and L1 and between $\mathrm{H} 2$ and L1, and up to $1 \mathrm{~ms}$ between $\mathrm{H} 1$ and $\mathrm{H} 2$ (to allow for a possible mismatch in time calibration). Specifically, $\Gamma$ is the average of 'confidence' values calculated from the absolute value of each of the three individual $r$-statistic values. A large value of $\Gamma$ indicates that the data streams are correlated to an extent that is highly unlikely to have resulted from normal instrumental noise fluctuations. This quantity complements $Z_{\mathrm{g}}$, providing a different and largely independent means for distinguishing real signals from background.

Figure 6 shows plots of $\Gamma$ versus $Z_{\mathrm{g}}$ for time-shifted triggers and for simulated gravitational-wave signals after the $\mathrm{H} 1 / \mathrm{H} 2$ amplitude consistency cut but before the $R_{0}$ cut. The time-shifted triggers with $\Gamma<12$ and $Z_{\mathrm{g}}<20$ are the tail of the bulk distribution of triggers. The outliers with $\Gamma>12$ all arise from a few distinct times when large noise transients occurred in $\mathrm{H} 1$ and $\mathrm{H} 2$; these are found many times, paired with different L1 time shifts, and have similar values of $\Gamma$ because the calculation of $\Gamma$ is dominated by the $\mathrm{H} 1-\mathrm{H} 2$ 


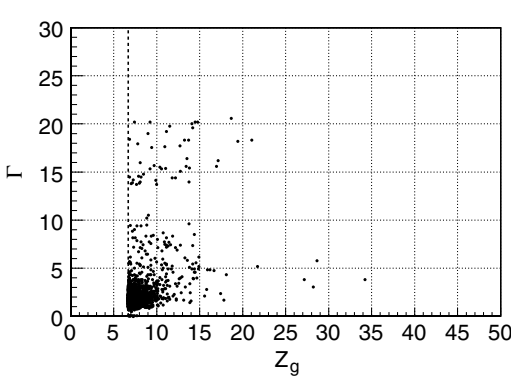

(a)

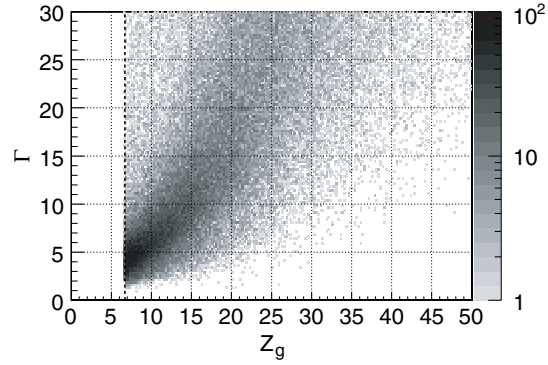

(b)

Figure 6. Plots of $\Gamma$ versus $Z_{\mathrm{g}}$, after the $\mathrm{H} 1 / \mathrm{H} 2$ amplitude consistency cut but before any other cuts. (a) Scatter plot for all time-shifted triggers in the tuning set. (b) A two-dimensional histogram, with bin count indicated by grayscale, for simulated sine-Gaussian signals with a wide range of frequencies and amplitudes from sources uniformly distributed over the sky (see section 7). In both the plots, the vertical dashed line indicates the initial WaveBurst significance cut at $Z_{\mathrm{g}}=6.7$.

pair in these cases. The outliers with $\Gamma<12$ and $Z_{\mathrm{g}}>20$ are artifacts of sudden changes in the power line noise at $60 \mathrm{~Hz}$ and $180 \mathrm{~Hz}$ which WaveBurst recorded as triggers. A cut on the value of $\Gamma$ can eliminate many of the time-shifted triggers in figure $6(a)$, but at the cost of also rejecting weak genuine gravitational-wave signals that may have the distribution in figure $6(b)$. Therefore, the $\Gamma$ cut is chosen only after additional selection criteria have been applied (see section 5.3).

\section{Additional selection criteria for event candidates}

Environmental disturbances or instrumental misbehaviour occasionally produce nonstationary noise in the GW channel of a detector which contributes to the recording of a WaveBurst trigger. These triggers can sometimes pass the $\mathrm{H} 1-\mathrm{H} 2$ consistency and crosscorrelation consistency tests, particularly since an environmental disturbance at the Hanford site affects both $\mathrm{H} 1$ and $\mathrm{H} 2$. As noted in the previous section, the calculated value of $\Gamma$ is susceptible to being dominated by the $\mathrm{H} 1-\mathrm{H} 2$ pair even if there is minimal signal power in the L1 data stream. A significant background rate of event candidates caused by environmental or instrumental effects could obscure the rare gravitational-wave bursts that we seek, or else require us to apply more aggressive cuts and thus lose sensitivity for weak signals.

This section describes the two general tactics that we use to reject data with identifiable problems and thereby reduce the rate of background triggers. First, we make use of several 'data quality flags' that have been introduced in order to describe the status of the instruments and the quality of the recorded data over time intervals ranging from seconds to hours. Second, we remove triggers attributed to short-duration instrumental or environmental effects by applying 'vetoes' based on triggers generated from auxiliary channels which have been found to correlate with transients in the GW channel. Applying data quality conditions and vetoes to the data set reduces the amount of 'live' observation time (or 'livetime') during which an arriving gravitational-wave burst would be detected and kept as an event candidate at the end of the analysis pipeline. Therefore, we must balance this loss ('deadtime') against the effectiveness for removing spurious triggers from the data sample.

Choosing data quality and veto conditions with reference to a sample of gravitationalwave event candidates could introduce a selection bias and invalidate any upper limit calculated 
from the sample. Therefore, we have evaluated the relevance of potential data quality cuts and veto conditions using other trigger samples. In addition to the tuning set of time-shifted WaveBurst triggers, we have applied the KleineWelle [27] method to identify transients in each interferometer's GW channel. (We have also used KleineWelle to identify transients in numerous auxiliary channels for veto studies, as described in section 5.2.) Like WaveBurst, KleineWelle is a time-frequency method utilizing multi-resolution wavelet decomposition, but it processes each data channel independently [28]. In analyzing data, the time series is first whitened using a linear predictor error filter [27]. Then the time-frequency decomposition is obtained using the Haar wavelet transform. The squared wavelet coefficients normalized to the scale's (frequency's) root mean square provide an estimate of the energy associated with a certain time-frequency pixel. A clustering mechanism is invoked in order to increase the sensitivity to signals with less than optimal shapes in the time-frequency plane and a total normalized cluster energy is computed. The significance of a cluster is then defined as the negative natural logarithm of the probability of the computed total normalized cluster energy to have resulted from Gaussian white noise; we apply a threshold on this significance to define KleineWelle triggers. The samples of KleineWelle triggers from each detector, as well as the subsample of coincident $\mathrm{H} 1$ and $\mathrm{H} 2$ triggers, are useful indicators of localized disturbances. They may, in principle, contain one or more genuine gravitational-wave signals, but decisions about data quality and veto conditions are based on the statistics of the entire sample which is dominated by instrumental artifacts and noise fluctuations.

\subsection{Data quality conditions}

We wish to reject instances of clear hardware problems with the LIGO detectors or conditions that could affect our ability to unequivocally register the passage of gravitational-wave bursts. Various studies of the data, performed during and after data collection, produced a catalog of conditions that might affect the quality of the data. Each named condition, or 'flag', has an associated list of time intervals during which the condition is present, derived either from one or more diagnostic channels or from entries made in the electronic logbook by operators and scientific monitors. We have looked for significant correlations between the flagged time intervals and time-shifted WaveBurst triggers, and also between the flagged time intervals and KleineWelle single-detector triggers (particularly, the 'outliers' with large significance and the coincident $\mathrm{H} 1$ and $\mathrm{H} 2$ triggers). Based on these studies, we decided to impose a number of data quality conditions.

We first require the calibration lines to be continuously present. On several occasions when they dropped out briefly, due to a problem with the excitation engine, the data are removed from the analysis. The livetime associated with these occurrences is negligible while they are all correlated with transients appearing in the GW channel.

Local winds and sound from airplanes may couple to the instrument through the ground and result in elevated noise and/or impulsive signals. A data quality flag was established to identify intervals of local winds at the sites with speeds of $56 \mathrm{~km} \mathrm{~h}^{-1}\left(35 \mathrm{miles} \mathrm{h}^{-1}\right)$ and above. We studied the correlation of these times with the single-detector triggers produced with KleineWelle. The correlation is more apparent in the $\mathrm{H} 2$ detector, for which $7.4 \%$ of the most significant KleineWelle triggers (threshold of 1600) coincide with the intervals of strong winds at the Hanford site. The livetime that is rejected in this way is $0.66 \%$ of the H1$\mathrm{H} 2$ coincident observation time over which this study was performed. Thanks to improved acoustic isolation installed after the S2 science run, acoustic noise from airplanes was not found to contribute to triggers in the GW channel in general; however, a period of $300 \mathrm{~s}$ has been rejected around a particularly loud time when a fighter jet passed over the Hanford site. 
Elevated low-frequency seismic activity has been observed to cause noise fluctuations and transients in the GW channel. Data from several seismometers at the Hanford observatory were band-pass filtered in various narrow bands between $0.4 \mathrm{~Hz}$ and $2.4 \mathrm{~Hz}$, and the root-meansquare signal in each band was tracked over time. A set of particularly relevant seismometers and bands was selected, and time intervals were flagged whenever a band in this set exceeded seven times its median value. A follow up analysis of the single instrument as well as coincident $\mathrm{H} 1-\mathrm{H} 2$ KleineWelle triggers found significant correlation with the elevated seismic noise. The strongest correlation is observed in the outlier triggers (KleineWelle significance of 1600 or greater) in $\mathrm{H} 2$, of which $41.9 \%$ coincide with the seismic flags, compared to a deadtime of $0.6 \%$.

In the two Hanford detectors, a diagnostic channel counting ADC overflows in the length sensing and control subsystem was used to flag intervals for exclusion from the analysis. One minute of livetime around these overflows is rejected. Such overflows were indeed seen to correlate with single-detector outlier triggers in $\mathrm{H} 1$ (44.4\% of them with $0.68 \%$ deadtime) and $\mathrm{H} 2$ ( $74.1 \%$ of them with $0.41 \%$ deadtime).

Two data quality cuts are derived from 'trend' data (summaries of minimum, maximum, mean and root-mean-square values over each $1 \mathrm{~s}$ period) monitoring the interferometry used in the LIGO detectors. The first one is based on occasional transient dips in the stored light in the arm cavities. These have been identified by scanning the trend data for the relevant monitoring photodiodes, defining the size of a dip as the fractional drop of the minimum in that second relative to the average of the previous $10 \mathrm{~s}$, and applying various thresholds on the minimum dip size. For the three LIGO detectors, thresholds of 5\%, 4\% and 5\% respectively for L1, H1 and $\mathrm{H} 2$ are used. High correlation of such light dips with single-detector triggers is observed, while the deadtime resulting from them in each of the three LIGO instruments is less than $0.6 \%$. The second data quality cut of this type is based on the DC level of light reaching the photodiode at the output of the interferometer, which sees very little light when the interferometer is operating properly. By thresholding on the trend data for this channel, intervals when its value was unusually high are identified in $\mathrm{H} 1$ and L1. These intervals are seen to correlate with instrument outlier triggers significantly. The deadtime resulting from them is $1.02 \%$ in $\mathrm{H} 1$ and $1.74 \%$ in $\mathrm{L} 1$.

Altogether, these data quality cuts result in a net loss of observation time of $5.6 \%$.

\subsection{Auxiliary-channel vetoes}

LIGO records thousands of auxiliary read-back channels of the servo control systems employed in the instruments' interferometric operation as well as auxiliary channels monitoring the instruments' physical environment. There are plausible couplings of environmental disturbances or servo instabilities both to these monitoring channels and to the GW channel; thus, transients appearing in these auxiliary channels may be used to veto triggers seen simultaneously in the GW channel. This assumes that a genuine gravitational-wave burst would not appear in these auxiliary channels, or at least that any coupling is small enough to stay below the threshold for selecting transients in these channels.

We have used KleineWelle to produce triggers from over 100 different auxiliary channels that monitor the interferometry and the environment in the three LIGO detectors. A first analysis of single-detector KleineWelle triggers from the L1 GW channel and coincident KleineWelle triggers from the $\mathrm{H} 1$ and $\mathrm{H} 2 \mathrm{GW}$ channels against respective auxiliary channels identified those that showed high GW channel trigger rejection power with minimal livetime loss (in the vast majority of channels much less that 1\%). In addition to interferometric channels, environmental ones (accelerometers and microphones) located on the optical tables 
holding the output optics and photodiodes appeared to correlate with GW channel triggers recorded at the same site.

Auxiliary interferometric channels (besides the GW channel) could, in principle, be affected by a gravitational wave, and a veto condition derived from such a channel could reject a genuine signal. Hardware signal injections imitating the passage of gravitational waves through our detectors, performed at several pre-determined times during the run, have been used to establish under what conditions each channel is safe to use as a veto. Non-detection of a hardware injection by an auxiliary channel suggests the unconditional safety of this channel as a veto in the search, assuming that a reasonably broad selection of signal strengths and frequencies were injected. But even if the hardware injections are seen in the auxiliary channels, conditions can readily be derived under which no triggers caused by the hardware injections are used as vetoes. This involves imposing conditions on the significance of the trigger and/or on the ratio of the signal strength seen in the auxiliary channel to that seen in the GW channel. We have thus established the conditions under which several channels involved in the length and angular sensing and control systems of the interferometers can be used safely as vetoes. (The data quality conditions described in section 5.1 were also verified to be safe using the hardware injections.)

The final choice of vetoes was made by examining the tuning set of time-shifted triggers remaining in the WaveBurst search pipeline after applying the signal consistency tests and data quality conditions. The ten triggers from the time-shifted analysis with the largest values of $\Gamma$, plus the ten with the largest values of $Z_{\mathrm{g}}$, were examined and six of them were found to coincide with transients in one or more of the following channels: the in-phase and quadraturephase demodulated signals from the pick-off beam from the H1 beamsplitter, the in-phase demodulated pitch signal from one of the wavefront sensors used in the $\mathrm{H} 1$ alignment sensing and control system, the beam splitter pitch and yaw control signals, and accelerometer readings on the optical tables holding the $\mathrm{H} 1$ and $\mathrm{H} 2$ output optics and photodiodes. KleineWelle triggers produced from these seven auxiliary channels were clustered (with a $250 \mathrm{~ms}$ window) and their union was taken. This defines the final list of veto triggers for this search, each indicating a time interval (generally, $\ll 1 \mathrm{~s}$ long) to be vetoed.

The total duration of the veto triggers considered in this analysis is at the level of $0.15 \%$ of the total livetime. However, this does not reliably reflect the deadtime of the search, since a GW channel trigger is vetoed if it has any overlap with a veto trigger. Thus, the actual deadtime of the search depends on the duration of the signal being sought, as reconstructed by WaveBurst. We reproduce this effect in the Monte Carlo simulation used to estimate the efficiency of the search (described in section 7) by applying the same analysis pipeline and veto logic. The effective deadtime depends on the morphology of the signal and on the signal amplitude, since larger-amplitude signals tend to be assigned longer durations by WaveBurst. For the majority of waveforms we considered in this search and for plausible signals strengths, the resulting effective deadtime is of the order of $2 \%$. Because this loss is signal dependent, in this analysis we consider it to be a loss of efficiency rather than a loss of live observation time; in other words, the live observation time we state reflects the data quality cuts applied but does not reflect the auxiliary-channel vetoes.

\subsection{Gamma cut}

The cuts described above cleaned up the outliers in the data considerably, as shown by the sequence of scatter plots in figure 7. Following the data quality and veto criteria we just described, the remaining time-shifted WaveBurst triggers (shown in figure $7(d)$ ) were used as the basis for choosing the cross-correlation $\Gamma$ threshold. As with previous all-sky searches for 


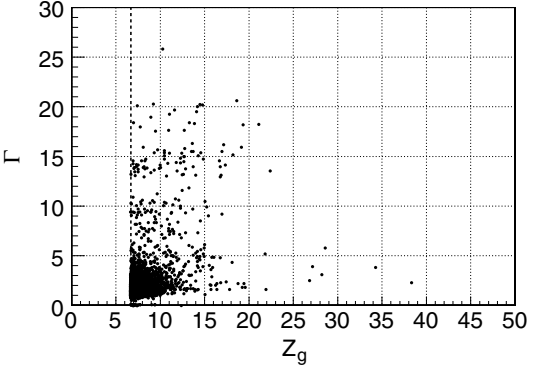

(a)

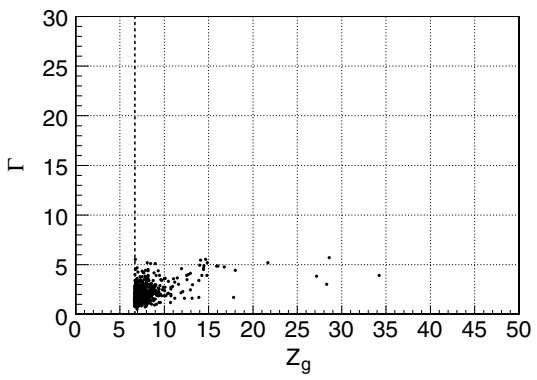

(c)

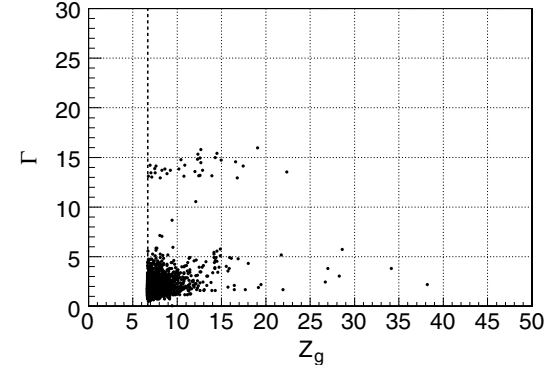

(b)

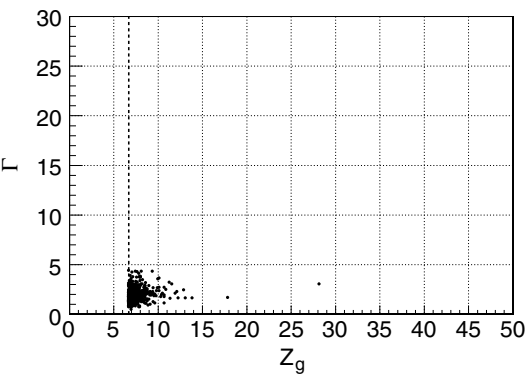

(d)

Figure 7. Scatter plots of $\Gamma$ versus $Z_{\mathrm{g}}$ for the tuning set of time-shifted triggers. (a) All triggers; (b) after data quality cuts; (c) after data quality and $\mathrm{H} 1-\mathrm{H} 2$ consistency cuts (amplitude ratio and $\left.R_{0}\right) ;(d)$ after data quality, $\mathrm{H} 1-\mathrm{H} 2$ consistency, and auxiliary-channel vetoes.

gravitational-wave bursts with LIGO, we desire the number of background triggers expected for the duration of the observation to be much less than 1 but not zero, typically of order $\sim 0.1$. On that basis, we chose a threshold of $\Gamma>4$ which results in seven triggers in 98 time shifts, or 0.08 such triggers normalized to the duration of the $\mathrm{S} 4$ observation time.

\section{Search results}

After all of the trigger selection criteria had been established using the tuning set of timeshifted triggers, WaveBurst was re-run with a new, essentially independent set of 100 time shifts, in increments of $5 \mathrm{~s}$ from $-250 \mathrm{~s}$ to $-5 \mathrm{~s}$ and from $+5 \mathrm{~s}$ to $+250 \mathrm{~s}$, in order to provide an estimate of the background which is minimally biased by the choice of selection criteria. The total effective livetime for the time-shifted sample is 77.4 times the unshifted observation time, reflecting the reduced overlap of Hanford and Livingston data segments when shifted relative to one another. The unshifted triggers were looked at for the first time. Table 1 summarizes the trigger counts for these time-shifted and unshifted triggers at each stage in the sequence of cuts. In addition, the expected background at each stage (time-shifted triggers normalized to the S4 observation time) is shown for direct comparison with the observed zero-lag counts. Figure 8 shows a scatter plot of $\Gamma$ versus $Z_{\mathrm{g}}$ and histograms of $\Gamma$ for both time-shifted and unshifted triggers after all other cuts. These new time-shifted triggers are statistically consistent with the tuning set (figure $7(d)$ ), although no triggers are found with $Z_{\mathrm{g}}>15$ in this case. Five unshifted triggers are found, distributed in a manner reasonably consistent with the background. All five have $\Gamma<4$ and thus fail the $\Gamma$ cut. Three time-shifted 


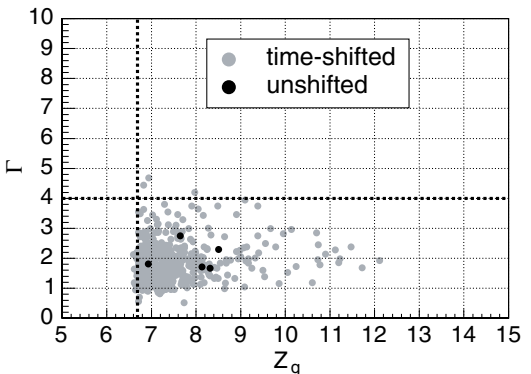

$(a)$

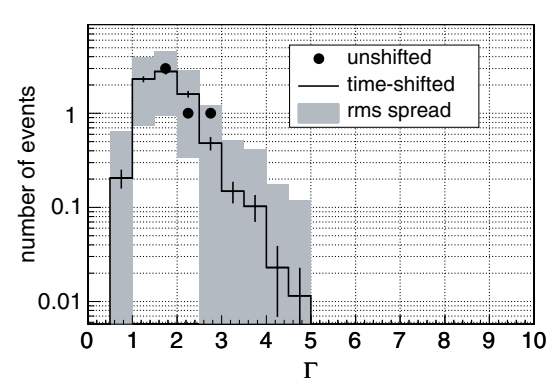

(b)

Figure 8. (a) Scatter plot of $\Gamma$ versus $Z_{\mathrm{g}}$ for time-shifted triggers (gray circles) and unshifted triggers (black circles) after all other analysis cuts. The vertical dashed line indicates the initial WaveBurst significance cut at $Z_{\mathrm{g}}=6.7$. The horizontal dashed line indicates the final $\Gamma$ cut. (b) Overlaid histograms of $\Gamma$ for unshifted triggers (black circles) and mean background estimated from time-shifted triggers (black stairstep with statistical error bars). The shaded bars represent the expected root-mean-square statistical fluctuations on the number of unshifted background triggers in each bin.

Table 1. Counts of time-shifted and unshifted triggers as cuts are applied sequentially. The column labeled 'normalized' is the time-shifted count divided by 77.4, representing an estimate of the expected background for the $\mathrm{S} 4$ observation time.

\begin{tabular}{lrll}
\hline & \multicolumn{2}{c}{ Time shifted } & Unshifted \\
\cline { 2 - 3 } Cut & Count & Normalized & count \\
\hline Data quality & 3153 & 40.7 & 44 \\
H1/H2 amplitude consistency & 1504 & 19.4 & 14 \\
$R_{0}>0$ & 755 & 9.8 & 5 \\
Auxiliary-channel vetoes & 671 & 8.7 & 5 \\
$\Gamma>4$ & 3 & 0.04 & 0 \\
\hline
\end{tabular}

triggers pass the $\Gamma$ cut, corresponding to an estimated average background of 0.04 triggers over the S4 observation time.

With no unshifted triggers in the final sample, we place an upper limit on the mean rate of gravitational-wave events that would be detected reliably (i.e., with efficiency near unity) by this analysis pipeline. Since the background estimate is small and is subject to some systematic uncertainties, we simply take it to be zero for purposes of calculating the rate limit; this makes the rate limit conservative. With 15.5 days of observation time, the one-sided frequentist upper limit on the rate at $90 \%$ confidence level is $-\ln (0.1) / T=2.303 /(15.5$ days $)=0.15$ day $^{-1}$. For comparison, the S2 search [17] arrived at an upper limit of 0.26 day $^{-1}$. The S3 search [18] had an observation time of only 8 days and did not state a rate limit.

\section{Amplitude sensitivity of the search}

The previous section presented a limit on the rate of a hypothetical population of gravitationalwave signals for which the analysis pipeline has perfect detection efficiency. However, the actual detection efficiency will depend on the signal waveform and amplitude, being zero for very weak signals and generally approaching unity for sufficiently strong signals. The signal 
processing methods used in this analysis are expressly designed to be able to detect arbitrary waveforms as long as they have short duration and frequency content in the $64-1600 \mathrm{~Hz}$ band which stands out above the detector noise. Therefore, for any given signal of this general type, we wish to determine a characteristic minimum signal amplitude for which the pipeline has good detection efficiency. As in past analyses, we use a Monte Carlo technique with a population of simulated gravitational-wave sources. Simulated events are generated at random sky positions and pseudo-random times (imposing a minimum separation of $80 \mathrm{~s}$ ) during the S4 run; the resulting signal waveforms in each interferometer are calculated with the appropriate antenna factors and time delays. These simulated signals are added to the actual detector data, and the summed data streams are analyzed using the same pipeline with the same trigger selection criteria.

The intrinsic amplitude of a simulated gravitational wave may be characterized by its root-sum-squared strain amplitude at the Earth, without folding in antenna response factors:

$$
h_{\mathrm{rss}} \equiv \sqrt{\int\left(\left|h_{+}(t)\right|^{2}+\left|h_{\times}(t)\right|^{2}\right) \mathrm{d} t} .
$$

This quantity has units of $\mathrm{s}^{1 / 2}$, or equivalently $\mathrm{Hz}^{-1 / 2}$. In general, the root-sum-squared signal measured by a given detector, $h_{\text {det }}^{\text {rss }}$, will be somewhat smaller. The Monte Carlo approach taken for this analysis is to generate a set of signals all with fixed $h_{\mathrm{rss}}$ and then to add this set of signals to the data with several discrete scale factors to evaluate different signal amplitudes. For a given signal morphology and $h_{\mathrm{rss}}$, the efficiency of the pipeline is the fraction of simulated signals which are successfully recovered.

For this analysis, we do not attempt to survey the complete spectrum of astrophysically motivated signals, but rather we use a limited number of ad hoc waveforms to characterize the sensitivity of the search in terms of $h_{\mathrm{rss}}$. Similar sensitivities may be expected for different waveforms with similar overall properties (central frequency, bandwidth, duration); the degree to which this is true has been investigated in $[18,29]$. The waveforms evaluated in the present analysis are:

- Sine-Gaussian: sinusoid with a given frequency $f_{0}$ inside a Gaussian amplitude envelope with dimensionless width $Q$ and arrival time $t_{0}$,

$$
h\left(t_{0}+t\right)=h_{0} \sin \left(2 \pi f_{0} t\right) \exp \left(-\left(2 \pi f_{0} t\right)^{2} / 2 Q^{2}\right) .
$$

These are generated with linear polarization, with $f_{0}$ ranging from $70 \mathrm{~Hz}$ to $1053 \mathrm{~Hz}$ and with $Q$ equal to 3,8.9 and 100. The signal consistency tests described in section 4 were developed using an ensemble of sine-Gaussian signals with all simulated frequencies and $Q$ values.

- Gaussian: a simple unipolar waveform with a given width $\tau$ and linear polarization,

$$
h\left(t_{0}+t\right)=h_{0} \exp \left(-t^{2} / \tau^{2}\right)
$$

- Band-limited white-noise burst: a random signal with two independent polarization components that are white over a given frequency band, described by a base frequency $f_{0}$ and a bandwidth $\Delta f$ (i.e. containing frequencies from $f_{0}$ to $f_{0}+\Delta f$ ). The signal amplitude has a Gaussian time envelope with a width $\tau$. Because these waveforms have two uncorrelated polarizations (in a coordinate system at some random angle), they provide a stringent check on the robustness of our cross-correlation test.

In all cases, we generate each simulated signal with a random arrival direction and a random angular relationship between the wave polarization basis and the Earth. 

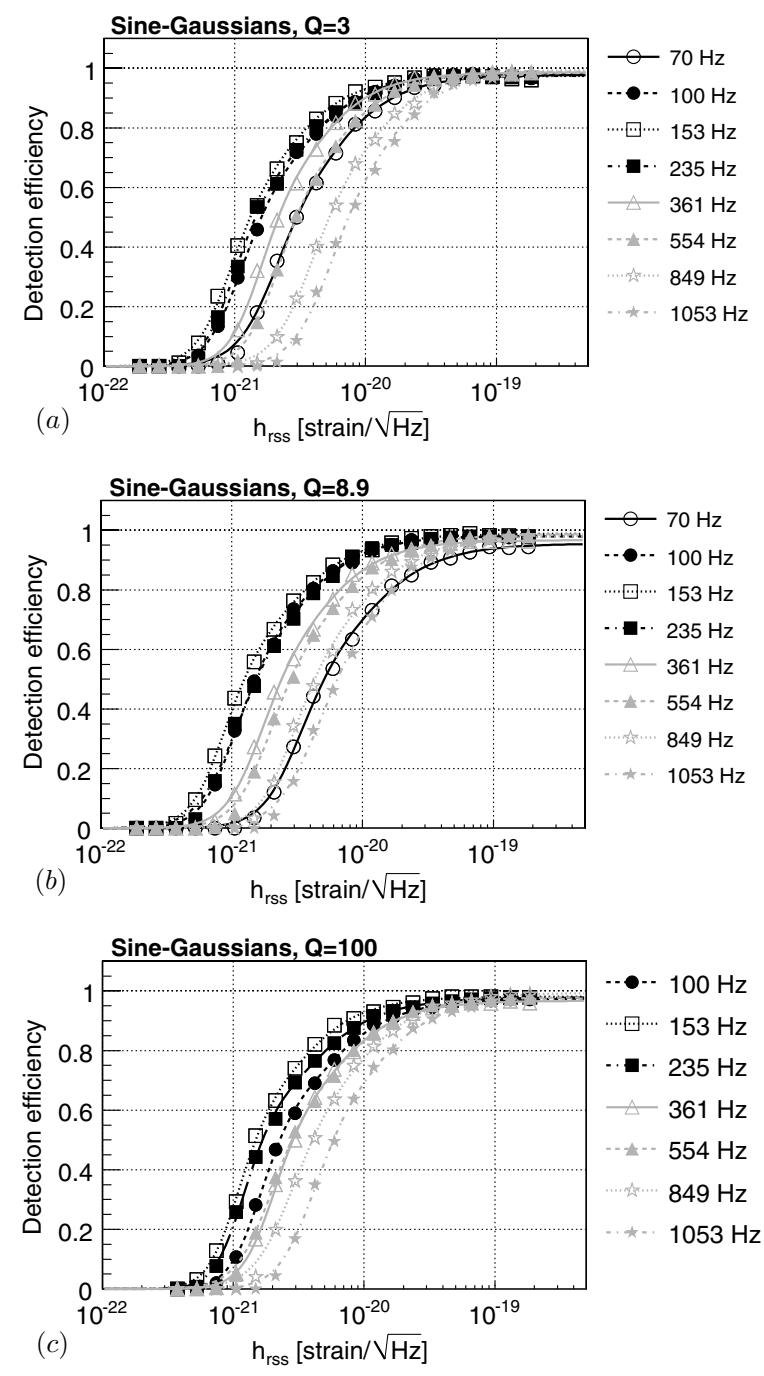

Figure 9. Efficiency curves for simulated gravitational-wave signals: linearly-polarized sineGaussian waves with (a) $Q=3$, (b) $Q=8.9$, (c) $Q=100$. Statistical errors are comparable to the size of the plot symbols.

Figures 9 and 10 show the measured efficiency of the analysis pipeline as a function of root-sum-squared strain amplitude, $\epsilon\left(h_{\mathrm{rss}}\right)$, for each simulated waveform. The efficiency data points for each waveform are fit with a function of the form

$$
\epsilon\left(h_{\mathrm{rss}}\right)=\frac{\epsilon_{\mathrm{max}}}{1+\left(\frac{h_{\mathrm{rss}}}{h_{\mathrm{rss}}^{\mathrm{mid}}}\right)^{\alpha\left(1+\beta \tanh \left(h_{\mathrm{rss}} / h_{\mathrm{rss}}^{\mathrm{mid}}\right)\right)},},
$$

where $\epsilon_{\max }$ corresponds to the efficiency for strong signals (normally very close to unity), $h_{\mathrm{rss}}^{\mathrm{mid}}$ is the $h_{\mathrm{rss}}$ value corresponding to an efficiency of $\epsilon_{\max } / 2, \beta$ is the parameter that describes the asymmetry of the sigmoid (with range -1 to +1 ) and $\alpha$ describes the slope. Data points with efficiency below 0.05 are excluded from the fit because they do not necessarily follow the functional form, while data points with efficiency equal to 1.0 are excluded because 

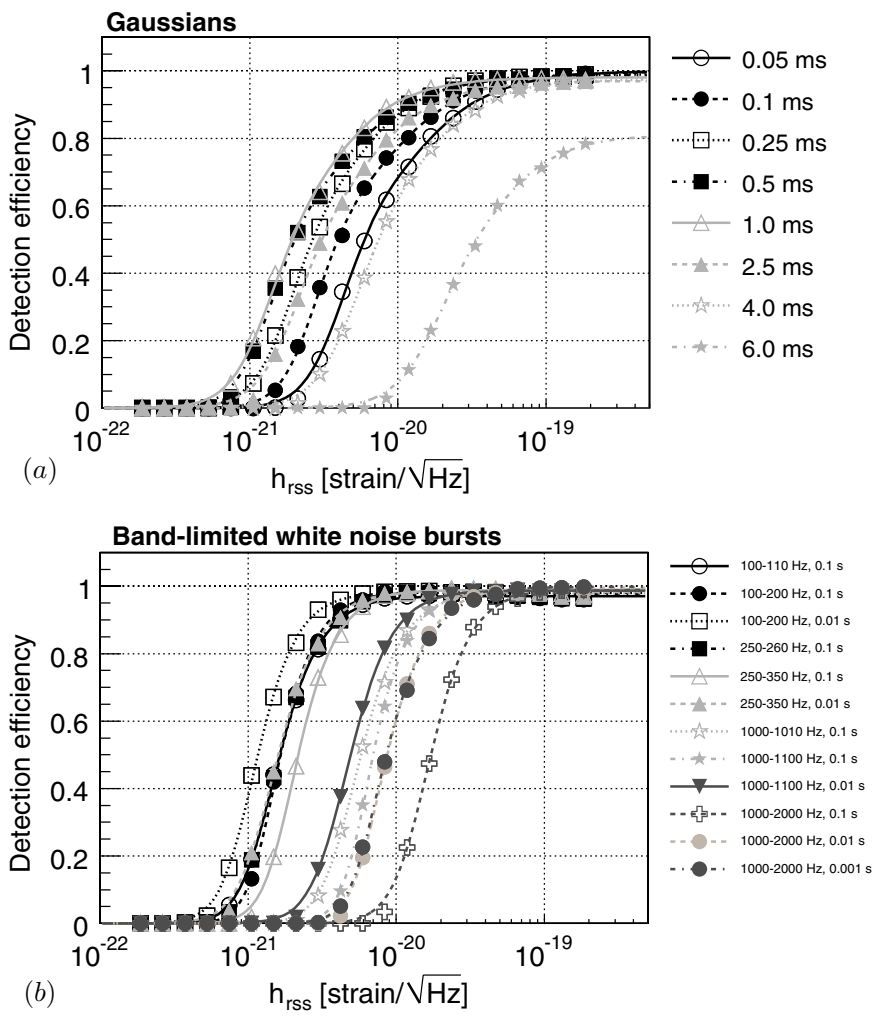

Figure 10. Efficiency curves for simulated gravitational-wave signals: (a) linearly-polarized Gaussian waves; (b) band-limited white-noise bursts with two independent polarization components. Note that four curves in the latter plot are nearly identical: $100-110 \mathrm{~Hz}, 0.1 \mathrm{~s}$; 100-200 Hz, $0.1 \mathrm{~s} ; 250-260 \mathrm{~Hz}, 0.1 \mathrm{~s}$; and 250-350 Hz, $0.01 \mathrm{~s}$. Statistical errors are comparable to the size of the plot symbols.

their asymmetric statistical uncertainties are not handled properly in the chi-squared fit. The empirical functional form in equation (7) has been found to fit the remaining efficiency data points well.

Note that the Gaussian waveform with $\tau=6.0 \mathrm{~ms}$ has efficiency less than 0.8 even for the largest simulated amplitude. This broad waveform, with little signal power at frequencies above $64 \mathrm{~Hz}$ (the lower end of the nominal search range), is at the limit of what the search method can detect. For some of the other waveforms, the efficiency levels off at a value slightly less than 1.0 due to the application of the auxiliary-channel vetoes, which randomly coincide in time with some of the simulated signals. This effect is most pronounced for the longest-duration simulated signals due to the veto logic used in this analysis, which rejects a trigger if there is any overlap between the reconstructed trigger duration and a vetoed time interval. The $70 \mathrm{~Hz}$ sine-Gaussian with $Q=100$ has a duration longer than $1 \mathrm{~s}$ and is reconstructed quite poorly; it is omitted from figure $9(c)$ and from the following results.

The analytic expressions of the fits are used to determine the signal strength $h_{\mathrm{rss}}$ for which efficiencies of $50 \%$ and $90 \%$ are reached. These fits are subject to statistical errors from the limited number of simulations performed to produce the efficiency data points. Also, the overall amplitude scale is subject to the uncertainty in the calibration of the interferometer 


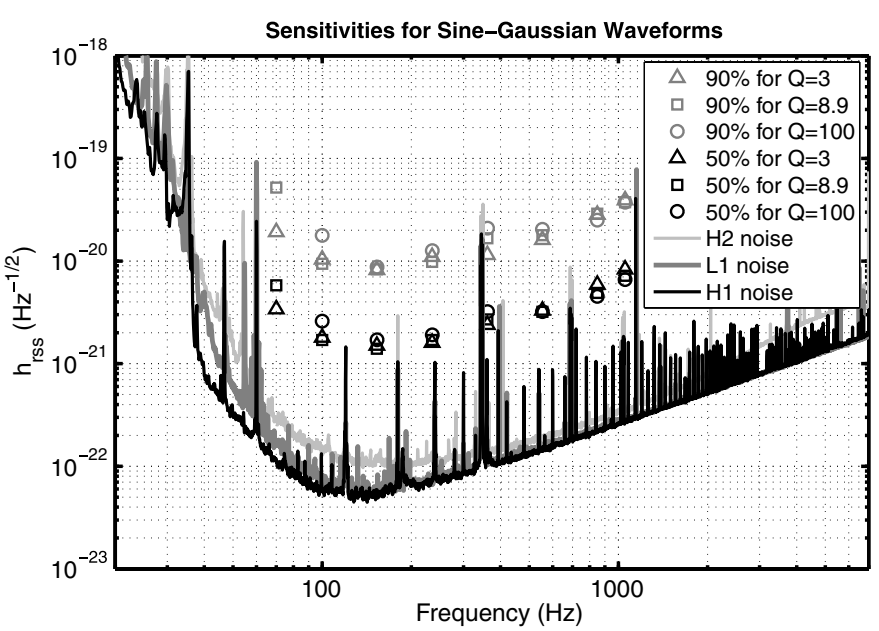

Figure 11. Sensitivity of the analysis pipeline for sine-Gaussian waveforms as a function of frequency and $Q$. Symbols indicate the $h_{\text {rss }}$ values corresponding to $50 \%$ and $90 \%$ efficiency, taken from table 2 . The instrumental sensitivity curves from figure 2 are shown for comparison.

Table 2. $h_{\mathrm{rss}}$ values corresponding to $50 \%$ and $90 \%$ detection efficiencies for simulated sineGaussian signals with various central frequencies and $Q$ values. The $70 \mathrm{~Hz}$ sine-Gaussian with $Q=100$ is not detected reliably.

\begin{tabular}{|c|c|c|c|c|c|c|}
\hline \multirow{3}{*}{$\begin{array}{l}\text { Central } \\
\text { frequency }(\mathrm{Hz})\end{array}$} & \multicolumn{6}{|c|}{$h_{\mathrm{rss}}\left(10^{-21} \mathrm{~Hz}^{-1 / 2}\right)$} \\
\hline & \multicolumn{3}{|c|}{$50 \%$ efficiency } & \multicolumn{3}{|c|}{$90 \%$ efficiency } \\
\hline & $Q=3$ & $Q=8.9$ & $Q=100$ & $Q=3$ & $Q=8.9$ & $Q=100$ \\
\hline 70 & 3.4 & 5.8 & - & 19.2 & 52.0 & - \\
\hline 100 & 1.8 & 1.7 & 2.6 & 10.4 & 9.4 & 17.7 \\
\hline 153 & 1.5 & 1.4 & 1.7 & 8.2 & 8.3 & 8.7 \\
\hline 235 & 1.6 & 1.7 & 1.9 & 11.0 & 9.8 & 12.6 \\
\hline 361 & 2.4 & 2.7 & 3.2 & 11.5 & 16.7 & 20.9 \\
\hline 554 & 3.3 & 3.2 & 3.2 & 16.1 & 17.9 & 20.4 \\
\hline 849 & 5.9 & 4.9 & 4.5 & 28.4 & 28.9 & 24.9 \\
\hline 1053 & 8.3 & 7.2 & 6.6 & 39.3 & 37.5 & 37.5 \\
\hline
\end{tabular}

response, conservatively estimated to be 10\% [30]. We increase the nominal fitted $h_{\mathrm{rss}}$ values by the amount of these systematic uncertainties to arrive at conservative $h_{\mathrm{rss}}$ values at efficiencies of $50 \%$ and $90 \%$, summarized in tables $2-4$. The sine-Gaussian $h_{\text {rss }}$ values are also displayed graphically in figure 11, showing how the frequency dependence generally follows that of the instrumental noise.

Event rate limits as a function of waveform type and signal amplitude can be represented by an 'exclusion diagram'. Each curve in an exclusion diagram indicates what the rate limit would be for a population of signals with a fixed $h_{\mathrm{rss}}$, as a function of $h_{\mathrm{rss}}$. The curves in figure 12 illustrate, using selected sine-Gaussian and Gaussian waveforms that were also considered in the S1 and S2 analyses, that the amplitude sensitivities achieved by this S4 analysis are at least an order of magnitude better than the sensitivities achieved by the S2 
Table 3. $h_{\mathrm{rss}}$ values corresponding to $50 \%$ and $90 \%$ detection efficiencies for simulated Gaussian signals with various widths. The waveform with $\tau=6.0 \mathrm{~ms}$ does not reach an efficiency of $90 \%$ within the range of signal amplitudes simulated.

\begin{tabular}{lcl}
\hline & \multicolumn{2}{c}{$h_{\mathrm{rss}}\left(10^{-21} \mathrm{~Hz}^{-1 / 2}\right)$} \\
\cline { 2 - 3 }$\tau(\mathrm{ms})$ & $50 \%$ efficiency & $90 \%$ efficiency \\
\hline 0.05 & 6.6 & 33.9 \\
0.1 & 4.4 & 25.3 \\
0.25 & 3.0 & 14.3 \\
0.5 & 2.2 & 13.5 \\
1.0 & 2.2 & 10.6 \\
2.5 & 3.4 & 20.5 \\
4.0 & 8.3 & 43.3 \\
6.0 & 39.0 & - \\
\hline
\end{tabular}

Table 4. $h_{\mathrm{rss}}$ values corresponding to $50 \%$ and $90 \%$ detection efficiencies for simulated 'whitenoise burst' signals with various base frequencies, bandwidths and durations.

\begin{tabular}{lclcc}
\hline \multirow{2}{*}{$\begin{array}{l}\text { Base frequency } \\
(\mathrm{Hz})\end{array}$} & $\begin{array}{c}\text { Bandwidth } \\
(\mathrm{Hz})\end{array}$ & $\begin{array}{l}\text { Duration } \\
(\mathrm{s})\end{array}$ & $50 \%$ efficiency & $90 \%$ efficiency \\
\hline 100 & 10 & 0.1 & 1.8 & 4.7 \\
100 & 100 & 0.1 & 1.9 & 4.1 \\
100 & 100 & 0.01 & 1.3 & 2.9 \\
250 & 10 & 0.1 & 1.8 & 4.5 \\
250 & 100 & 0.1 & 2.4 & 5.4 \\
250 & 100 & 0.01 & 1.8 & 4.3 \\
1000 & 10 & 0.1 & 6.5 & 15.8 \\
1000 & 100 & 0.1 & 7.9 & 16.7 \\
1000 & 100 & 0.01 & 5.5 & 12.7 \\
1000 & 1000 & 0.1 & 19.2 & 42.6 \\
1000 & 1000 & 0.01 & 9.7 & 22.3 \\
1000 & 1000 & 0.001 & 9.5 & 23.7 \\
\hline
\end{tabular}

analysis. For instance, the $50 \%$ efficiency $h_{\text {rss }}$ value for $235 \mathrm{~Hz}$ sine-Gaussians with $Q=8.9$ is $1.5 \times 10^{-20} \mathrm{~Hz}^{-1 / 2}$ for $\mathrm{S} 2$ and $1.7 \times 10^{-21} \mathrm{~Hz}^{-1 / 2}$ for $\mathrm{S} 4$. (Exclusion curves were not generated for the $\mathrm{S} 3$ analysis, but the $\mathrm{S} 3$ sensitivity was $9 \times 10^{-21} \mathrm{~Hz}^{-1 / 2}$ for this particular waveform.) The improvement is greatest for lower-frequency sine-Gaussians and for the widest Gaussians, due to the reduced low-frequency detector noise and the explicit extension of the search band down to $64 \mathrm{~Hz}$.

\section{Astrophysical reach estimates}

In order to set an astrophysical scale to the sensitivity achieved by this search, we can ask what amount of mass converted into gravitational-wave burst energy at a given distance would be strong enough to be detected by the search pipeline with $50 \%$ efficiency. We start with the expression for the instantaneous energy flux emitted by a gravitational-wave source in the two 

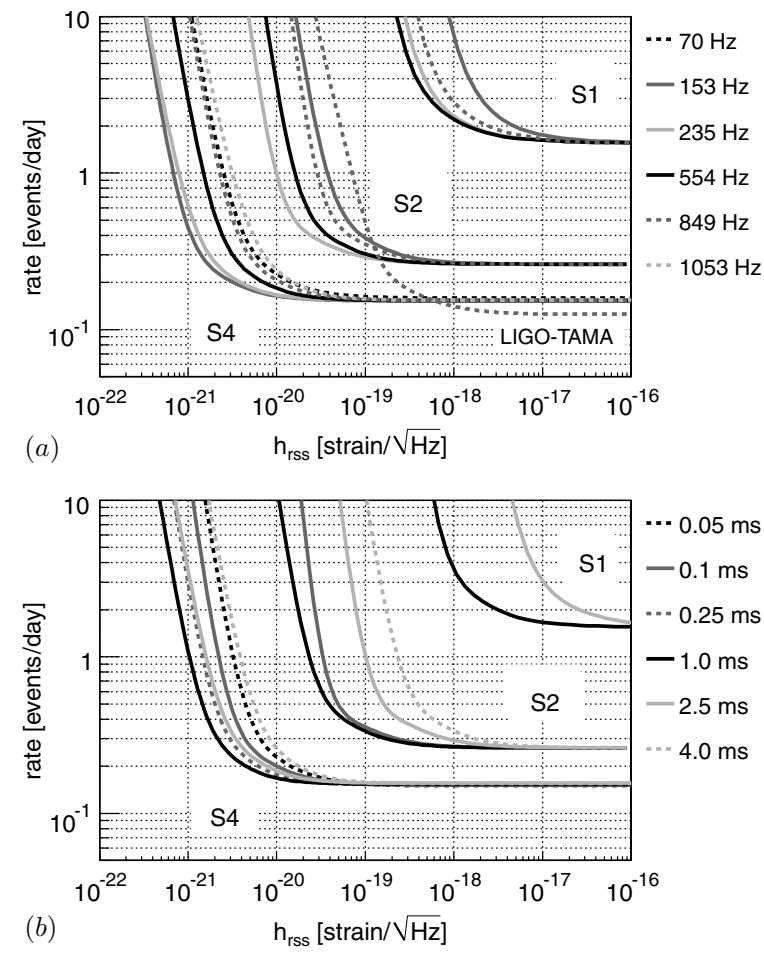

Figure 12. Exclusion diagrams (rate limit at $90 \%$ confidence level, as a function of signal amplitude) for (a) sine-Gaussian and (b) Gaussian-simulated waveforms for this S4 analysis compared to the S1 and S2 analyses (the S3 analysis did not state a rate limit). These curves incorporate conservative systematic uncertainties from the fits to the efficiency curves and from the interferometer response calibration. The $849 \mathrm{~Hz}$ curve labeled 'LIGO-TAMA' is from the joint burst search using LIGO S2 with TAMA DT8 data [8], which included data subsets with different combinations of operating detectors with a total observation time of 19.7 days and thereby achieved a lower rate limit. The $h_{\text {rss }}$ sensitivity of the LIGO-TAMA search was nearly constant for sine-Gaussians over the frequency range $700-1600 \mathrm{~Hz}$. (a) Sine-Gaussians with $Q=8.9$. (b) Gaussians.

independent polarizations $h_{+}(t)$ and $h_{\times}(t)[31]$

$$
\frac{\mathrm{d}^{2} E_{\mathrm{GW}}}{\mathrm{d} A \mathrm{~d} t}=\frac{1}{16 \pi} \frac{c^{3}}{G}\left\langle\left(\dot{h}_{+}\right)^{2}+\left(\dot{h}_{\times}\right)^{2}\right\rangle
$$

and follow the derivations in [32]. Plausible astrophysical sources will, in general, emit gravitational waves anisotropically, but here we will assume isotropic emission in order to get simple order-of-magnitude estimates. The above formula, when integrated over the signal duration and over the area of a sphere at radius $r$ (assumed not to be at a cosmological distance), yields the total energy emitted in gravitational waves for a given signal waveform. For the case of a sine-Gaussian with frequency $f_{0}$ and $Q \gg 1$, we find

$$
E_{\mathrm{GW}}=\frac{r^{2} c^{3}}{4 G}\left(2 \pi f_{0}\right)^{2} h_{\mathrm{rss}}^{2} .
$$

Taking the waveform for which we have the best $h_{\text {rss }}$ sensitivity, a $153 \mathrm{~Hz}$ sine-Gaussian with $Q=8.9$, and assuming a typical Galactic source distance of $10 \mathrm{kpc}$, the above formula relates 
the $50 \%$ efficiency $h_{\text {rss }}=1.4 \times 10^{-21} \mathrm{~Hz}^{-1 / 2}$ to $10^{-7}$ solar mass equivalent emission into a gravitational-wave burst from this hypothetical source and under the given assumptions. For a source in the Virgo galaxy cluster, approximately $16 \mathrm{Mpc}$ away, the same $h_{\text {rss }}$ would be produced by an energy emission of roughly $0.25 M_{\odot} c^{2}$ in a burst with this highly favorable waveform.

We can draw more specific conclusions about detectability for models of astrophysical sources which predict the absolute energy and waveform emitted. Here we consider the corecollapse supernova simulations of Ott et al [15] and a binary black hole merger waveform calculated by the Goddard numerical relativity group [11] (as a representative example of the similar merger waveforms obtained by several groups). While the Monte Carlo sensitivity studies in section 7 did not include these particular waveforms, we can relate the modeled waveforms to qualitatively similar waveforms that were included in the Monte Carlo study and thus infer the approximate sensitivity of the search pipeline for these astrophysical models.

Ott et al simulated core collapse for three progenitor models and calculated the resulting gravitational-wave emission, which was dominated by oscillations of the protoneutron star core driven by accretion [15]. Their s11WW model, based on a non-spinning $11 M_{\odot}$ progenitor, produced a total gravitational-wave energy emission of $1.6 \times 10^{-8} M_{\odot} c^{2}$ with a characteristic frequency of $\sim 654 \mathrm{~Hz}$ and duration of several hundred milliseconds. If this were a sineGaussian, it would have a $Q$ of several hundred; table 2 shows that our sensitivity does not depend strongly on $Q$, so we might expect $50 \%$ efficiency for a signal at this frequency with $h_{\mathrm{rss}}$ of $\sim 3.7 \times 10^{-21} \mathrm{~Hz}^{-1 / 2}$. However, the signal is not monochromatic, and its increased time-frequency volume may degrade the sensitivity by up to a factor of $\sim 2$. Using this $E_{\mathrm{GW}}$ and $h_{\mathrm{rss}} \approx 7 \times 10^{-21} \mathrm{~Hz}^{-1 / 2}$ in equation (9), we find that our search has an approximate 'reach' (distance for which the signal would be detected with $50 \%$ efficiency by the analysis pipeline) of $\sim 0.2 \mathrm{kpc}$ for this model. The m15b6 model, based on a spinning $15 M_{\odot}$ progenitor, yields a very similar waveform and essentially the same reach. The s $25 \mathrm{WW}$ model, based on a $25 M_{\odot}$ progenitor, was found to emit vastly more energy in gravitational waves, $8.2 \times 10^{-5} M_{\odot} c^{2}$, but with a higher characteristic frequency of $\sim 937 \mathrm{~Hz}$. With respect to the Monte Carlo results in section 7, we may consider this similar to a high-Q sine-Gaussian, yielding $h_{\mathrm{rss}} \approx 5.5 \times 10^{-21} \mathrm{~Hz}^{-1 / 2}$, or to a white-noise burst with a bandwidth of $\sim 100 \mathrm{~Hz}$ and a duration of $>0.1 \mathrm{~s}$, yielding $h_{\mathrm{rss}} \approx 8 \times 10^{-21} \mathrm{~Hz}^{-1 / 2}$. Using the latter, we deduce an approximate reach of $8 \mathrm{kpc}$ for this model.

A pair of merging black holes emits gravitational waves with very high efficiency; for instance, numerical evolutions of equal-mass systems without spin have found the radiated energy from the merger and subsequent ringdown to be $3.5 \%$ or more of the total mass of the system [11]. From figure 8 of that paper, the frequency of the signal at the moment of peak amplitude is seen to be

$$
f_{\text {peak }} \approx \frac{0.46}{2 \pi M_{f}} \approx \frac{15 \mathrm{kHz}}{\left(M_{f} / M_{\odot}\right)},
$$

where $M_{f}$ is the final mass of the system. Very roughly, we can consider the merger + ringdown waveform to be similar to a sine-Gaussian with central frequency $f_{\text {peak }}$ and $Q \approx 2$ for purposes of estimating the reach of this search pipeline for binary black hole mergers. (Future analyses will include Monte Carlo efficiency studies using complete inspiral-mergerringdown waveforms.) Thus, a binary system of two $10 M_{\odot}$ black holes (i.e. $M_{f} \approx 20 M_{\odot}$ ) has $f_{\text {peak }} \approx 750 \mathrm{~Hz}$, and from table 2 we can estimate the $h_{\text {rss }}$ sensitivity to be $\sim 5.5 \times 10^{-21} \mathrm{~Hz}^{-1 / 2}$. Using $E_{\mathrm{G} W}=0.035 M_{f} c^{2}$, we conclude that the reach for such a system is roughly $1.4 \mathrm{Mpc}$. Similarly, a binary system with $M_{f}=100 M_{\odot}$ has $f_{\text {peak }} \approx 150 \mathrm{~Hz}$, a sensitivity of $\sim 1.5 \times$ $10^{-21} \mathrm{~Hz}^{-1 / 2}$, and a resulting reach of roughly $60 \mathrm{Mpc}$. 


\section{Discussion}

The search reported in this paper represents the most sensitive search to date for gravitationalwave bursts in terms of strain amplitude, reaching $h_{\text {rss }}$ values below $10^{-20} \mathrm{~Hz}^{-1 / 2}$, and covers a broad frequency range, 64-1600 Hz, with a live observation time of 15.5 days.

Comparisons with previous LIGO [16, 17] and LIGO-TAMA [8] searches have already been shown graphically in figure 12. The LIGO-TAMA search targeted millisecond-duration signals with frequency content in the $700-2000 \mathrm{~Hz}$ frequency regime (i.e., partially overlapping the present search) and had a detection efficiency of at least 50\% (90\%) for signals with $h_{\mathrm{rss}}$ greater than $\sim 2 \times 10^{-19} \mathrm{~Hz}^{-1 / 2}\left(10^{-18} \mathrm{~Hz}^{-1 / 2}\right)$. Among other searches with broad-band interferometric detectors [33-35], the most recent one by the TAMA collaboration reported an upper limit of 0.49 events day ${ }^{-1}$ at the $90 \%$ confidence level based on an analysis of 8.1 days of the TAMA300 instrument's ninth data taking run (DT9) in 2003-2004. The best sensitivity of this TAMA search was achieved when looking for narrow-band signals at TAMA's best operating frequency, around $1300 \mathrm{~Hz}$, and it was at $h_{\mathrm{rss}} \approx 10^{-18} \mathrm{~Hz}^{-1 / 2}$ for $50 \%$ detection efficiency [35]. Although we did not measure the sensitivity of the S4 LIGO search with narrow-band signals at $1300 \mathrm{~Hz}$, LIGO's noise at that frequency range varies slowly enough so that we do not expect it to be significantly worse than the sensitivity for $1053 \mathrm{~Hz}$ sine-Gaussian signals described in section 7, which stands at about $7 \times 10^{-21} \mathrm{~Hz}^{-1 / 2}$.

Comparisons with results from resonant mass detectors were detailed in our previous publications $[16,17]$. The upper limit of $\sim 4 \times 10^{-3}$ events day ${ }^{-1}$ at the $95 \%$ confidence level on the rate of gravitational wave bursts set by the IGEC consortium of five resonant mass detectors still represents the most stringent rate limit for $h_{\mathrm{rss}}$ signal strengths of order $10^{-18} \mathrm{~Hz}^{-1 / 2}$ and above [36]. This upper limit quickly falls off and becomes inapplicable to signals weaker than $10^{-19} \mathrm{~Hz}^{-1 / 2}$ (see figure 14 in [17]). Furthermore, with the improvement in our search sensitivity, the signal strength of the events corresponding to the slight excess seen by the EXPLORER and NAUTILUS resonant mass detectors in their 2001 data [37] falls well above the $90 \%$ sensitivity of our current S4 search: as described in [17], the optimal orientation signal strength of these events assuming a Gaussian morphology with $\tau=0.1 \mathrm{~ms}$ corresponds to a $h_{\text {rss }}$ of $1.9 \times 10^{-19} \mathrm{~Hz}^{-1 / 2}$. For such Gaussians our S4 search all-sky $90 \%$ sensitivity is $2.5 \times 10^{-20} \mathrm{~Hz}^{-1 / 2}$ (see table 3 ) and when accounting for optimal orientation, this improves by roughly a factor of 3 , to $9.3 \times 10^{-21} \mathrm{~Hz}^{-1 / 2}$. The rate of the EXPLORER and NAUTILUS events was of order 200 events year $^{-1}$ (or 0.55 events day $^{-1}$ ) [37, 38]. A steady flux of gravitational-wave bursts at this rate is excluded by our present measurement at the 99.9\% confidence level. Finally, in more recent running of the EXPLORER and NAUTILUS detectors, an analysis of 149 days of data collected in 2003 set an upper limit of 0.02 events day $^{-1}$ at the $95 \%$ confidence level and with a $h_{\text {rss }}$ sensitivity of $\sim 2 \times 10^{-19} \mathrm{~Hz}^{-1 / 2}$ [39].

The S5 science run, which began in November 2005 and is expected to continue until late 2007, has a goal of collecting a full year of coincident LIGO science-mode data. Searches for gravitational-wave bursts using S5 data are already underway and will be capable of detecting any sufficiently strong signals which arrive during that time, or else placing an upper limit on the rate of such signals on the order of a few per year. Furthermore, the detector noise during the S5 run has reached the design goals for the current LIGO interferometers, and so the amplitude sensitivity of S5 burst searches is expected to be roughly a factor of two better than the sensitivity of this S4 search.

Another direction being pursued with the S5 data is to make appropriate use of different detector network configurations. In addition to the approach used in the S4 analysis reported here, which requires a signal to appear with excess power in a time-frequency map in all three LIGO interferometers, data from two-detector combinations is also being analyzed 
to maximize the total observation time. Furthermore, using LIGO data together with simultaneous data from other interferometers can significantly improve confidence in a signal candidate and allow more properties of the signal to be deduced. The GEO 600 interferometer has joined the S5 run for full-time observing in May 2006, and we look forward to the time when VIRGO begins operating with sensitivity comparable to the similarly-sized LIGO interferometers. Members of the LSC are currently implementing coherent network analysis methods using maximum likelihood approaches for optimal detection of arbitrary burst signal (see, for example, [40]) and for robust signal consistency tests [41, 42]. Such methods will make the best use of the data collected from the global network of detectors to search for gravitational-wave bursts.

\section{Acknowledgments}

The authors gratefully acknowledge the support of the United States National Science Foundation for the construction and operation of the LIGO Laboratory and the Science and Technology Facilities Council of the United Kingdom, the Max-Planck-Society, and the State of Niedersachsen/Germany for support of the construction and operation of the GEO 600 detector. The authors also gratefully acknowledge the support of the research by these agencies and by the Australian Research Council, the Council of Scientific and Industrial Research of India, the Istituto Nazionale di Fisica Nucleare of Italy, the Spanish Ministerio de Educaciòn y Ciencia, the Conselleria d'Economia, Hisenda i Innovaciò of the Govern de les Illes Balears, the Scottish Funding Council, the Scottish Universities Physics Alliance, The National Aeronautics and Space Administration, the Carnegie Trust, the Leverhulme Trust, the David and Lucile Packard Foundation, the Research Corporation, and the Alfred P Sloan Foundation. This document has been assigned LIGO Laboratory document number LIGO-P060016-B-Z.

\section{References}

[1] Sigg D (for the LSC) 2006 Class. Quantum Grav. 23 S51-6

[2] Lück H et al 2006 Class. Quantum Grav. 23 S71-8

[3] Acernese F et al 2006 Class. Quantum Grav. 23 S63-9

[4] Ando M and the TAMA Collaboration 2005 Class. Quantum Grav. 22 S881-9

[5] Fritschel P 2003 Gravitational-Wave Detection: Proc. SPIE vol 4856 ed M Cruise and P Saulson (Bellingham, WA: SPIE Optical Engineering Press) pp 282-91

[6] Acernese F et al 2006 Class. Quantum Grav. 23 S635-42

[7] Kuroda K et al 2003 Proc. 28th Int. Cosmic Ray Conf. ed T Kajita (Tokyo: Universal Academy) p 3103

[8] Abbott B et al (LSC) and Akutsu T et al (TAMA Collaboration) 2006 Phys. Rev. D 73102002

[9] Blanchet L, Damour T, Esposito-Farèse G and Iyer B R 2004 Phys. Rev. Lett. 93091101

[10] Jaranowski P, Królak A and Schutz B F 1998 Phys. Rev. D 58063001

[11] Baker J G, Centrella J, Choi D, Koppitz M and van Meter J 2006 Phys. Rev. D 73104002

[12] Dimmelmeier H, Font J A and Müller E 2001 Astrophys. J. Lett. 560 L163-6

[13] Ott C D, Burrows A, Livne E and Walder R 2004 Astrophys. J. 600 834-64

[14] Burrows A, Livne E, Dessart L, Ott C D and Murphy J 2006 Astrophys. J. 640 878-90

[15] Ott C D, Burrows A, Dessart L and Livne E 2006 Phys. Rev. Lett. 96201102

[16] Abbott B et al (LSC) 2004 Phys. Rev. D 69102001

[17] Abbott B et al (LSC) 2005 Phys. Rev. D 72062001

[18] Abbott B et al (LSC) 2006 Class. Quantum Grav. 23 S29-39

[19] Lazzarini A and Weiss R 1995 LIGO Science Requirements Document (SRD) LIGO technical document LIGOE950018-02-E

[20] Klimenko S and Mitselmakher G 2004 Class. Quantum Grav. 21 S1819-30 
[21] The version of WaveBurst used for this analysis may be found at http://ldas-sw.ligo.caltech.edu/cgibin/cvsweb.cgi/Analysis/WaveBurst/S4/?cvsroot=GDS with the CVS tag 'S4'

[22] Daubechies I 1992 Ten Lectures on Wavelets (Philadelphia, PA: SIAM)

[23] Klimenko S, Yakushin I, Rakhmanov M and Mitselmakher G 2004 Class. Quantum Grav. 21 S1685-94

[24] Cadonati L and Márka S 2005 Class. Quantum Grav. 22 S1159-67

[25] Cadonati L 2004 Class. Quantum Grav. 21 S1695-703

[26] The version of CorrPower used for this analysis may be found at http://www.lsc-group.phys.uwm.edu/cgibin/cvs/viewcvs.cgi/matapps/src/searches/burst/CorrPower/?cvsroot=lscsoft with the CVS tag 'CorrPower-080605'

[27] Chatterji S, Blackburn L, Martin G and Katsavounidis E 2004 Class. Quantum Grav. 21 S1809-18

[28] The version of KleineWelle used for this analysis may be found at http://ldas-sw.ligo.caltech.edu/cgibin/cvsweb.cgi/gds/Monitors/kleineWelle/?cvsroot=GDS dated January 20, 2005

[29] Beauville F et al 2006 A comparison of methods for gravitational wave burst searches from LIGO and Virgo, submitted to Class. Quantum Grav. (Preprint gr-qc/0701026)

[30] Dietz A, Garofoli J, González G, Landry M, O’Reilly B and Sung M 2006 LIGO technical document LIGOT050262-01-D

[31] Shapiro S L and Teukolsky S A 1983 Black Holes, White Dwarfs, and Neutron Stars (New York: Wiley)

[32] Riles K 2004 LIGO technical document LIGO-T040055-00-Z

[33] Nicholson D et al 1996 Phys. Lett. A 218175

[34] Forward R L 1978 Phys. Rev. D 17379

[35] Ando M et al (TAMA Collaboration) 2005 Phys. Rev. D 71082002

[36] Astone P et al (International Gravitational Event Collaboration) 2003 Phys. Rev. D 68022001

[37] Astone P et al 2002 Class. Quantum Grav. 195449

[38] Coccia E, Dubath F and Maggiore M 2004 Phys. Rev. D 70084010

[39] Astone P et al 2006 Class. Quantum Grav. 23 S169-78

[40] Klimenko S, Mohanty S, Rakhmanov M and Mitselmakher G 2005 Phys. Rev. D 72122002

[41] Wen L and Schutz B 2005 Class. Quantum Grav. 22 S1321-35

[42] Chatterji S, Lazzarini A, Stein L, Sutton P J, Searle A and Tinto M 2006 Phys. Rev. D 74082005 